\title{
Gestión de los bosques tropicales estacionalmente secos de la provincia de Santa Elena, Ecuador: una perspectiva desde la conservación
}

RECIBIDO: 18/02/2019 ACEPTADO: 07/06/2019

Evelyng Astudillo-SÁNChez ${ }^{1}$ JAME PÉREZ FLOR ${ }^{2}$ GILMER MEDina ${ }^{3}$ Ana Medina ${ }^{4}$

\begin{abstract}
RESUMEN
Los bosques tropicales estacionalmente secos son reconocidos por su importancia biológica y económica al poseer un alto número de especies endémicas y proveer diversos servicios ecosistémicos. No obstante, la carencia de una planificación para la conservación de la biodiversidad amenaza la gestión ambiental integral en las áreas protegidas de la provincia de Santa Elena, Ecuador. Por ello, se realizó un diagnóstico en el que se identificaron los objetos de conservación para fortalecer la capacidad de manejo ambiental local. Asimismo, se emplearon las metodologías de Planificación para la Conservación de Áreas (PCA) y Fortalezas, Oportunidades, Debilidades y Amenazas (FODA) para conocer las prioridades de conservación y las limitaciones en el manejo de los recursos. En el diagnóstico se evidenciaron falencias técnico-administrativas, debido a ello se buscó un enfoque eficiente en la gestión ambiental. Además, se destacaron el bosque de garúa como objeto natural y el cabildo como objeto cultural para conservar. La gobernanza ambiental participativa debe ser fortalecida para mejorar la gestión de las áreas protegidas.
\end{abstract}

Palabras-claves: Áreas protegidas; bosques tropicales estacionalmente secos; gestión ambiental local; Santa Elena (Ecuador).

\section{INTRODUCCIÓN}

Una herramienta legal para la conservación de la naturaleza ha sido la creación de áreas protegidas. A nivel mundial, el establecimiento y manejo de las áreas protegidas es la estrategia más empleada para la conservación de ecosistemas y su biodiversidad (Chávez et al., 2014). Sin embargo, esta estrategia global necesita acompañarse de una planificación e integrarla a una matriz de territorio con aspectos socioeconómicos y políticos que requieren de trabajos pluridisciplinarios (Gurrutxaga y Lozano, 2009). Las áreas protegidas no están exentas de problemas de conservación debido a la existencia de presencia humana, ya sea dentro o en la periferia del territorio. La deforestación como problema es un reto en la gestión de las áreas protegidas y es una de las principales fuentes de emisión de $\mathrm{CO}_{2}$ que contribuye al cambio climático (CC), además de ser la segunda principal amenaza que enfrenta la biodiversidad.

El efecto de la deforestación provoca que un ecosistema se fragmente en parches, y que estos no alberguen gran diversidad de especies debido al efecto de borde, afectando las redes de interacción tróficas (EUROPARC-España, 2009). La Organización de las Naciones Unidas para la Alimentación (2016) registró para la región tropical, en el periodo $2000-2010$, una pérdida de cobertura forestal de siete millones de hectáreas y un incremento anual neto de superficie agrícola de seis millones de hectáreas.

En Ecuador, el Sistema Nacional de Áreas Protegidas (SNAP) se estableció en 1959 con la creación del Parque Nacional Galápagos; luego, en 1996, se constituyó el Ministerio del Ambiente

1 Bióloga por la Universidad de Guayaquil, Ecuador. Magíster en Espacios Naturales Protegidos por la Universidad Autónoma de Madrid. Investigadora Asociada al Research Center, Universidad Espíritu Santo, Samborondón, Ecuador.

ORCID: 0000-0003-0006-2730

E-mail: eveavesecuador@gmail.com

2 Biólogo por la Universidad de Guayaquil, Ecuador. Actualmente es Director del Jardín Botánico de Guayaquil y consultor independiente.

E-mail:jperez_40@hotmail.com

3 Geográfo por la Universidad Nacional Mayor de San Marcos y Mágister en Ciencias Ambientales por la Universidad Nacional Mayor de San Marcos. Actualmente es docente de la Facultad de Ingeniería de la Universidad Privada del Norte y Decano del Colegio de Geógrafos del Perú. Lima, Perú.

E-mail: gilmer.medina@upn.edu.pe

4 Ingeniera Química por la Universidad Nacional Mayor de San Marcos. Docente de la Facultad de Ingeniería Industrial de la Universidad Nacional Mayor de San Marcos.

E-mail: amedinae@unmsm.edu.pe 
como autoridad ambiental y único administrador del SNAP (Columba, 2013). En 2008, la política ambiental en el país evolucionó hacia un enfoque biocéntrico con la nueva constitución y el sumak kawsay, filosofía indígena de cosmovisión, en la cual se reconoce la plurinacionalidad e interculturalidad del Estado, se otorgan derechos al medio ambiente y se concede autonomía territorial (Altmann, 2013). Así se involucró a la sociedad en la gestión de las áreas protegidas y se estructuró al SNAP en cuatro subsistemas: 1) estatal- Patrimonio de Áreas Naturales del Estado (PANE), 2) autónomo descentralizado-Gobiernos Autónomos Descentralizados (GAD), 3) comunitario-indígenas y afroecuatorianos y 4) privado (Columba, 2013).

La provincia de Santa Elena protege el 28\% de su extensión terrestre, mientras que el $4 \%$ forma parte del subsistema comunitario (Ministerio del Ambiente del Ecuador, 2017). A pesar de esto, ha sido la provincia, junto con Guayas, la más afectada por la deforestación durante el periodo 2000-2008 (Ministerio del Ambiente del Ecuador, 2012a). La deforestación se ha venido reportando desde la década de 1990 por autores como Dodson y Gentry, Parker y Carr, Bonifaz y Cornejo, Astudillo, Pérez y Fabara, entre otros, quienes confirman que el área ha sido perturbada por la extracción selectiva, mientras que en algunos sitios el bosque ha sido convertido en zonas de pasto para el ganado.

Los ecosistemas presentes en la provincia de Santa Elena (PSE) forman parte de los bosques tropicales estacionalmente secos (BTES) del Pacífico ecuatorial, los cuales conforman una región florística que posee una alta diversidad y endemismos; no obstante, es considerado uno de los ecosistemas tropicales más amenazados debido al nivel de alteración y fragmentación (Best y Kessler, 1995).

El aporte económico de los bosques secos es conocido, pero no cuantificado, ya que carecen de investigación, particularmente en la PSE, y sus pocas contribuciones realizadas en Ecuador se han enfocado concretamente en el sur. Además, los bosques secos han sido subestimados, según Aguirre y Erazo (2017), quienes resaltan la importancia de estudios en valoración ecológica y económica de estos bosques, pues brindan una diversidad de servicios ecosistémicos. Un ejemplo de esto es el estudio en la comuna Loma Alta realizado por Becker (1999), investigación que se centra en la hipótesis de que los comuneros parecen no apreciar los valores indirectos del bosque de tierras altas, el cual les proporciona el suministro de agua con el que subsisten y el riego en la agricultura.
La interdependencia entre el bienestar humano y la provisión de servicios ecosistémicos de los bosques está en aumento; por ello, es necesario garantizar, a largo plazo, el funcionamiento de estos, donde las áreas protegidas jueguen un rol esencial en la conservación de los recursos naturales y culturales. La planificación y el manejo eficiente de estos recursos son un reto para los administradores de las áreas protegidas, quienes operan una serie de elementos de carácter legal, administrativo, social, financiero, entre otros (Cifuentes et al., 2000).

Es imprescindible contar con una estrategia de planificación para la conservación de los BTES del Pacífico ecuatorial ubicados en la Cordillera Chongón-Colonche ( $\mathrm{CCHC}$ ) y conocer los valores naturales y culturales con el fin de conservarlos como primer paso. Por ello, el objetivo de esta investigación es identificar los objetos de conservación presentes en dos áreas protegidas ubicadas en la provincia de Santa Elena para la elaboración de una estrategia de desarrollo local y fortalecer la capacidad de manejo ambiental.

\section{METODOLOGÍA}

\section{Área de estudio}

Aspectos geopolíticos. La PSE posee una extensión de $3762,8 \mathrm{~km}^{2}$ y está dividida geopolíticamente por tres cantones: Santa Elena $\left(3668,9 \mathrm{~km}^{2}\right)$, Salinas $\left(68,7 \mathrm{~km}^{2}\right)$ y La Libertad $\left(25,3 \mathrm{~km}^{2}\right)$ (ver figura 1), donde Santa Elena es el cantón con mayor actividad agropecuaria, destinando el $14 \%$ de su superficie para cultivos y $6 \%$ para pastizal. Este cantón es el que posee, también, mayores recursos forestales, ya que al año 2008 indicaba una superficie de $31 \%$ como bosque nativo y $42 \%$ como vegetación arbustiva, mostrando un uso del suelo notable para la conservación (Gobierno Autónomo Descentralizado Municipal Santa Elena, 2014). En el cantón Santa Elena se eligieron dos localidades con áreas protegidas: la comuna Loma Alta $\left(01^{\circ} 52^{\prime} \mathrm{S}, 80^{\circ} 38^{\prime} \mathrm{O}\right)$ (ver figura 1 ), la cual posee un bosque protector con 3218,19 ha, que es conocido como Reserva Ecológica Comunal Loma Alta; y, la comuna Dos Mangas ( $\left.02^{\circ} 07^{\prime} \mathrm{S}, 80^{\circ} 18^{\prime} \mathrm{O}\right)$ (ver figura 1), que forma parte del bosque protector Chongón-Colonche y posee una superficie de 83731 ha (Acción en el Biocorredor, 2012).

Aspectos biogeográficos. CCHC atraviesa la PSE en su extremo noreste (ver figura 1); además, presenta un clima variable influenciado por las corrientes marinas y el gradiente altitudinal. La corriente fría de Humboldt provoca un efecto de neblina y llovizna conocida como garúa de junio a noviembre, 

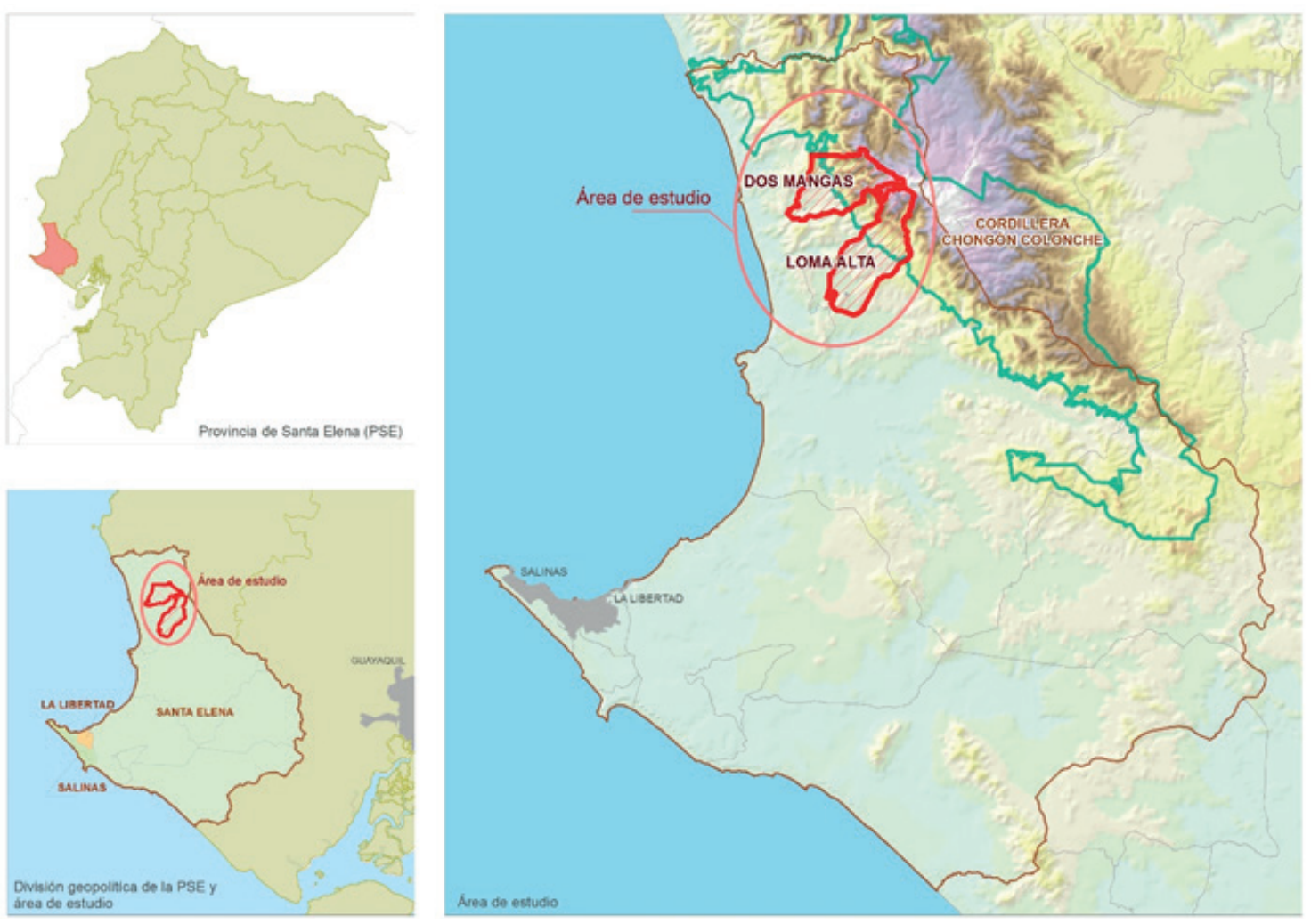

Figura 1. Ubicación geográfica de las comunas Loma Alta y Dos Mangas, PSE.

Fuente: elaboración propia.

la cual aporta el $40 \%$ de agua que el bosque, a partir de 400 m s. n. m., intercepta (Becker, 1999). La PSE presenta dos estaciones marcadas: una lluviosa, que va de diciembre a abril, y otra seca, que va de junio a octubre, la cual coincide con los meses de garúa. En el cantón Santa Elena, la temperatura promedio interanual es de $23,4^{\circ} \mathrm{C}$ y su precipita-

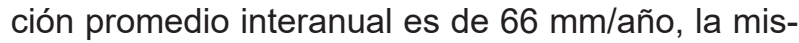
ma que presenta una variabilidad hasta de $200 \mathrm{~mm} /$ mes en la estación lluviosa y cercana a cero en la estación seca (Gobierno Autónomo Descentralizado Municipal Santa Elena, 2014).

En $\mathrm{CCHC}$ convergen dos regiones biogeográficas: Chocó y Tumbes. Actualmente, fusionadas y reconocidas formalmente como la ecorregión terrestre del Chocó-Darien-Ecuador occidental, que se extiende desde la «parte sureste de Panamá, a lo largo de las partes occidentales de Colombia y Ecuador, hasta el noroeste del Perú» (Critical Ecosystem Partnership Fund, 2005, p. 6). A esta ecorregión terrestre se la conoce como el hotspot Tumbes-Chocó-Magdalena, el cual está caracterizado por una diversidad de ecosistemas, desde bosques húmedos premontanos hasta matorrales espinosos secos.
En términos florísticos, $\mathrm{CCHC}$ forma parte del sector cordillera costera del Pacífico ecuatorial (CCPE), la misma que comprende las partes altas y piedemontes de la Chongón-Colonche con un bioclima de semiárido a subhúmedo, donde la vegetación es característica de ecosistemas húmedo a partir de 400 m s. n. m. y secos en las partes bajas (Ministerio del Ambiente del Ecuador, 2012b). En la cordillera costera del Pacífico ecuatorial, se identificaron cuatro ecosistemas: 1) bosque deciduo de la CCPE, asentado en el piedemonte de los flancos y colinas bajas de la $\mathrm{CCHC}$, en un piso bioclimático de $>200 \mathrm{~m} \mathrm{~s}$. n. m., con un clima xérico-seco y estrato arbóreo entre 15 y 20 m de altura con especies caducas; 2) bosque semideciduo de la CCPE, encontrado en las crestas y laderas, en un piso bioclimático $>200 \mathrm{~m} \mathrm{~s}$. $\mathrm{n}$. $\mathrm{m}$., con clima pluviestacional-subhúmedo y estrato arbóreo entre 12 y $25 \mathrm{~m}$ de altura con el $25 \%$ de especies caducifolias; 3) bosque siempreverde estacional piemontano de la CCPE, ubicado en un piso bioclimático de 200-400 m s. n. m., con clima pluviestacional-húmedo y estrato arbóreo promedio de $20 \mathrm{~m}$ con árboles emergentes hasta $30 \mathrm{~m}$ de altura; 4) bosque siempreverde estacional montano bajo de la CCPE, localizado en un piso bioclimático de $400-860 \mathrm{~m}$ s. n. m., con clima pluviestacional-húmedo y bosque multiestratificado 
de árboles con hasta $25 \mathrm{~m}$ de altura (Ministerio del Ambiente del Ecuador, 2012b).

Aspectos socioeconómicos. Los habitantes poseen una identidad cultural propia de los pueblos precolombinos, tales como Valdivia (4200-1500 a. C.), Manteño-Guancavilca y Milagro-Quevedo (periodo de integración 800 d. C.-1535), los cuales marcaron étnicamente la dinámica sociocultural establecida por jefaturas complejas (López y Peralta, 2016). Según la Ley de Comunas promulgada en 1936: «los descendientes de estos pueblos precolombinos han conservado cierta estructura social y económica, congregándose en comunidades indígenas y campesinas [...], la cual garantiza el ejercicio de los derechos colectivos indígenas o afroecuatorianos que se autodefinen con nacionalidades de raíces ancestrales» (Astudillo, 2010, p. 22).

\section{METODOLOGÍA}

La metodología de planificación elegida fue el modelo de diseño para la conservación creado por The Nature Conservancy (TNC), que posee cuatro componentes: 1) planificación de la conservación ecorregional, donde se seleccionan áreas conocidas como portafolios de conservación; 2) planificación para la conservación de sitios o desarrollo de estrategias, ya sea en sitios individuales o en una red de áreas protegidas; 3 ) acción de conservación o toma de decisiones, donde se define el tipo de apoyo; y, 4) medición del éxito, donde se evalúan las acciones (Granizo et al., 2006).

Acorde al objetivo planteado, esta investigación se enfoca en el segundo componente del modelo diseño para la conservación; aunque se debe destacar que parte de los resultados del primer componente se exponen aquí al seleccionar nuestra área de estudio, pues forman parte del portafolio de conservación para la PSE.

Para el desarrollo de estrategias, se requirió conocer las prioridades de conservación dentro de cada localidad; y, por ello, se empleó como herramienta la metodología de Planificación para la Conservación de Áreas (PCA), que incluye una fase de diagnóstico del área, cuyo primer paso es la selección de los objetos de conservación (Granizo et al., 2006). El diagnóstico, además, se complementó con la técnica de análisis FODA para determinar las potencialidades y limitaciones en el manejo, gestión y uso público de los recursos naturales y culturales de las comunas y sus áreas protegidas. El análisis FODA es una herramienta de planificación estratégica que evalúa los factores fuertes y débiles al interior de una organización o proyecto, así como las oportunidades y amenazas de su ambiente exterior y se presenta en una matriz base (Ponce, 2006).

El análisis FODA y la selección de los objetos de conservación se efectuaron mediante cuatro talleres participativos durante los años 2015, 2016 y 2017, con un aproximado de veinte participantes por cada taller, también se involucró a expertos locales, actores sociales y representantes de organizaciones ambientales gubernamentales y no gubernamentales.

Los objetos de conservación son elementos o características únicas encontradas a diferentes niveles de organización biológica y escalas geográficas que permiten identificar los sitios de conservación para una ecorregión o las amenazas y desarrollar estrategias para un área (Groves et al., 2000). Debido al objetivo del proyecto y la logística en las áreas, se decidió realizar el primer taller con un grupo multi y transdisciplinario de expertos en el área de la ingeniería (ingenieros ambientales, forestales, civiles, mecánicos, turísticos) y biólogos, quienes ayudaron con el objetivo inicial de diseñar el portafolio de conservación para la PSE. Los siguientes talleres fueron in situ con las comunidades $y$, mediante la técnica lluvia de ideas, se enlistaron objetos de conservación naturales $(\mathrm{OCN})$ y objetos de conservación culturales (OCC).

Acorde a las categorías y a los requisitos descritos por Granizo et al. (2006), se escogieron un total de ocho elementos en cada localidad, cuatro para cada tipo de objeto, y se empleó la técnica de filtro grueso-filtro fino para ayudarnos con la definición de los objetos. Esta técnica consiste en una pirámide invertida, donde se especifican cuatro escalas espaciales (regional, gruesa, intermedia y local) y se describen tres niveles de organización biológica (especies, comunidades y ecosistemas) (Groves et al., 2000). Lo que se presume con la utilización del filtro grueso-filtro fino, de acuerdo con Granizo et al. (2006), es que mediante el filtro grueso se protejan las comunidades y ecosistemas estratégicos que garanticen la funcionalidad y viabilidad de estos; mientras que, en el filtro fino, se conservarían aquellas especies que se encuentran en su interior o se escapan a la protección.

\section{RESULTADOS}

El análisis FODA se organizó en áreas temáticas: el desarrollo económico local, desarrollo social y capital humano, desarrollo institucional y participación ciudadana, así como el ordenamiento territorial y desarrollo de infraestructuras. Véase la tabla 1. 
Tabla 1. Matriz FODA de las comunas Loma Alta y Dos Mangas y sus áreas protegidas.

\begin{tabular}{|c|c|c|c|}
\hline Fortalezas & Oportunidades & Debilidades & Amenazas \\
\hline $\begin{array}{l}\text { Presencia de recursos natura- } \\
\text { les y culturales en las comunas }\end{array}$ & $\begin{array}{l}\text { Potencial para la investigación } \\
\text { científica en diversas áreas }\end{array}$ & $\begin{array}{l}\text { Sobrecarga de funciones del } \\
\text { cabildo }\end{array}$ & $\begin{array}{l}\text { Desaprovechamiento de las } \\
\text { oportunidades }\end{array}$ \\
\hline $\begin{array}{c}\text { Provisión de servicios ecosis- } \\
\text { témicos por parte de las áreas } \\
\text { protegidas }\end{array}$ & $\begin{array}{c}\text { Potencial para implementar di- } \\
\text { versos mecanismos financieros } \\
\text { innovadores para la conserva- } \\
\text { ción de la biodiversidad como } \\
\text { Pagos por Servicios Ambienta- } \\
\text { les (PSA) }\end{array}$ & $\begin{array}{l}\text { Falta de experiencia, moti- } \\
\text { vación y formación por parte } \\
\text { de la comunidad para iniciar } \\
\text { emprendimientos y desarrollo } \\
\text { de actividades en el sector eco- } \\
\text { nómico, turístico y ambiental }\end{array}$ & $\begin{array}{l}\text { Crisis económica actual que } \\
\text { afronta el país para sustentar } \\
\text { programas establecidos }\end{array}$ \\
\hline $\begin{array}{c}\text { Altos niveles de biodiversidad y } \\
\text { endemismo }\end{array}$ & $\begin{array}{l}\text { Apoyo del Ministerio del Am- } \\
\text { biente (MAE) para que el área } \\
\text { protegida se anexe al PANE }\end{array}$ & $\begin{array}{c}\text { Carencia de asesoramiento } \\
\text { técnico continuo en temas de } \\
\text { planificación, gestión y desarro- } \\
\text { llo comunitario }\end{array}$ & \multirow{2}{*}{$\begin{array}{l}\text { Incremento de problemas } \\
\text { ambientales como el cambio } \\
\text { climático, pérdida de cobertura } \\
\text { vegetal natural, degradación } \\
\text { de la cuenca hidrográfica, } \\
\text { fragmentación de hábitat y } \\
\text { contaminación de los recursos } \\
\text { naturales }\end{array}$} \\
\hline $\begin{array}{l}\text { Presentan una declaratoria } \\
\text { legal del área protegida como } \\
\text { bosque protector }\end{array}$ & $\begin{array}{l}\text { Potencial para crear una marca } \\
\text { ambiental con productos deri- } \\
\text { vados de la biodiversidad }\end{array}$ & $\begin{array}{l}\text { Falta mayor autogestión por } \\
\text { parte de la comunidad }\end{array}$ & \\
\hline $\begin{array}{c}\text { Pertenecen al programa Socio } \\
\text { Bosque y reciben un incentivo } \\
\text { económico anual para la ges- } \\
\text { tión de recursos }\end{array}$ & $\begin{array}{l}\text { Apoyo del GAD municipal y } \\
\text { provincial de Santa Elena }\end{array}$ & $\begin{array}{c}\text { Recursos financieros muy } \\
\text { limitados para programas de } \\
\text { conservación, pese a recibir } \\
\text { un incentivo económico de } \\
\text { Socio Bosque dedicado a este } \\
\text { objetivo }\end{array}$ & $\begin{array}{l}\text { Dependencia económica casi } \\
\text { exclusiva hacia el programa de } \\
\text { Socio Bosque para administrar } \\
\text { los recursos naturales }\end{array}$ \\
\hline $\begin{array}{c}\text { Declaratoria como Área de Im- } \\
\text { portancia para la Conservación } \\
\text { de Aves (AICAS) en ambas } \\
\text { comunas }\end{array}$ & $\begin{array}{l}\text { Potencial de iniciativas de } \\
\text { turismo comunitario sustentable } \\
\text { en diversas modalidades }\end{array}$ & $\begin{array}{l}\text { Limitado conocimiento del } \\
\text { idioma inglés para atender la } \\
\text { demanda de turismo interna- } \\
\text { cional }\end{array}$ & $\begin{array}{l}\text { Insuficiente reconocimiento, va- } \\
\text { loración y promoción nacional } \\
\text { del área protegida }\end{array}$ \\
\hline $\begin{array}{l}\text { Interés por parte del cabildo, } \\
\text { guardabosques y otros actores } \\
\text { claves en mejorar la planifica- } \\
\text { ción y manejo de sus recursos } \\
\text { naturales y culturales }\end{array}$ & $\begin{array}{c}\text { Cuenta con el apoyo de las } \\
\text { ONG ambientalistas nacionales } \\
\text { e internacionales }\end{array}$ & $\begin{array}{c}\text { Carencia de una estructura } \\
\text { administrativa y dirección es- } \\
\text { tratégica que administre el área } \\
\text { protegida }\end{array}$ & $\begin{array}{l}\text { Limitada cultura organizativa y } \\
\text { liderazgo para iniciar emprendi- } \\
\text { mientos económicos y sociales }\end{array}$ \\
\hline $\begin{array}{l}\text { Creciente interés del turismo } \\
\text { ecológico en áreas protegidas } \\
\text { boscosa en la PSE }\end{array}$ & $\begin{array}{c}\text { Creciente reconocimiento del } \\
\text { área protegida a nivel interna- } \\
\text { cional gracias a los esfuerzos } \\
\text { de investigación y divulgación } \\
\text { de los recursos }\end{array}$ & \multirow{2}{*}{$\begin{array}{l}\text { Loma Alta no posee un grupo } \\
\text { de interpretadores ambientales } \\
\text { comunitarios para continuar } \\
\text { con actividades turísticas } \\
\text { guiadas }\end{array}$} & $\begin{array}{c}\text { Ubicación geográfica de la co- } \\
\text { muna Loma Alta y difícil acceso } \\
\text { al área protegida }\end{array}$ \\
\hline $\begin{array}{l}\text { Predisposición de los jóvenes } \\
\text { comuneros para capacitarse } \\
\text { y desarrollar actividades de } \\
\text { turismo sostenible }\end{array}$ & $\begin{array}{l}\text { Posibilidad de establecer alian- } \\
\text { zas estratégicas con universi- } \\
\text { dades }\end{array}$ & & $\begin{array}{l}\text { Competencia en la oferta de } \\
\text { productos turísticos en la PSE }\end{array}$ \\
\hline $\begin{array}{l}\text { Alto nivel de seguridad ciuda- } \\
\text { dana }\end{array}$ & $\begin{array}{l}\text { Existen eventos ambientales } \\
\text { (p. e., conteo navideño) y cultu- } \\
\text { rales (p. e., sabores y saberes) } \\
\text { establecidos }\end{array}$ & \multirow{2}{*}{$\begin{array}{c}\text { Escaso nivel de educación } \\
\text { superior y carencia de conoci- } \\
\text { mientos por parte de los líderes } \\
\text { comunitarios para el manejo del } \\
\text { área protegida }\end{array}$} & $\begin{array}{c}\text { Restricciones para el acceso } \\
\text { vial y de telecomunicaciones } \\
\text { entre el poblado y el área } \\
\text { protegida }\end{array}$ \\
\hline $\begin{array}{l}\text { Existencia de una legislación } \\
\text { ambiental estable }\end{array}$ & $\begin{array}{c}\text { Promoción turística por parte } \\
\text { de la campaña All you need } \\
\text { is Ecuador, del Ministerio de } \\
\text { Turismo }\end{array}$ & & $\begin{array}{l}\text { Incremento de problemas } \\
\text { sociales por falta de trabajo, } \\
\text { carencia de servicios médicos, } \\
\text { de planificación familiar y edu- } \\
\text { cación (secundaria y superior) }\end{array}$ \\
\hline $\begin{array}{l}\text { Disponibilidad de superficie } \\
\text { para la creación de nueva } \\
\text { infraestructura }\end{array}$ & $\begin{array}{l}\text { Elaboración actual de un plan } \\
\text { de manejo participativo para } \\
\text { Loma Alta }\end{array}$ & $\begin{array}{l}\text { Falta de solidaridad entre los } \\
\text { comuneros }\end{array}$ & $\begin{array}{l}\text { Presencia de conflictos de } \\
\text { intereses de ciertos actores } \\
\text { sociales }\end{array}$ \\
\hline
\end{tabular}

Fuente: elaboración propia. 
Basado en el diagnóstico y en el análisis FODA, a continuación se presenta información relevante para el desarrollo de una estrategia local y se proponen varias acciones necesarias para conservar los recursos, asegurar la integridad ecológica y fortalecer la capacidad de gestión ambiental local.

En el ámbito técnico, la elaboración de un plan de manejo para cada área protegida es imperativo, ya que contendrá una propuesta de zonificación, donde los diferentes beneficiarios alcancen la compatibilidad de sus intereses en zonas funcionales especiales para su actividad productiva, así como áreas de conservación. Se sugiere que se actualice la normativa de aprovechamiento de los recursos naturales y se especifique los mecanismos que aseguren su cumplimiento.

En el ámbito administrativo, la creación de una unidad administrativa con personal calificado estimulará la participación e incentivará la economía a nivel local. La unidad debe contar con director, técnicos, asistentes y guardabosques, inicialmente hasta que se consolide y se extienda acorde a sus necesidades.

En el ámbito socio-educativo, la participación social y educación ambiental con criterios de diversidad, inclusión y equidad que empodere a los actores y sectores para una adecuada planificación y gestión de los recursos es trascendental. El desarrollo de la investigación científica multi y transdisciplinaria mediante proyectos y programas de monitoreos a largo plazo son primordiales para apoyar la toma de decisiones.

En el ámbito socio-político, la gobernanza presenta un modelo de cogestión entre la administración pública representada por el Ministerio del Ambiente del Ecuador (MAE) a través de sus respectivas direcciones, los gobiernos autónomos descentralizados (por ejemplo, gobernación y municipalidad), y la administración local constituida por el cabildo en cada comunidad. Sin embargo, carece de una planificación integral del territorio que envuelva el concepto de sostenibilidad con interacciones sociales horizontales, sistematizadas y multiniveles. Por lo tanto, se necesita una reingeniería en los procesos de gestión ambiental participativa que se unifique en una política local, nacional y regional.

En el ámbito económico, la diversificación de mecanismos financieros es urgente para que se integre al proceso de cambio en la matriz productiva enfocada en la agricultura. Los mecanismos financieros innovadores basados en los servicios ambientales propuestos se relacionan al pago por el uso o impacto en servicios ambientales y culturales, así como manejo de la biodiversidad y los recursos no maderables.

Por otra parte, también en los talleres se identificaron un total de ocho objetos de conservación, cuatro naturales y cuatro culturales, coincidiendo estos en ambas comunidades y con la lista preliminar de los expertos. Los objetos de conservación naturales (OCN) referidos fueron: 1) bosque de garúa; 2) sistemas hidrológicos; 3) especies cinegéticas que asocian a este grupo al venado de cola blanca (Odocoileus virginianus), saíno (Tayassu tajaco), guanta (Agouti paca), guatusa (Dasyprocta punctata), puesto que existe una tradición de uso consuntivo local de estas especies; y, 4) avifauna, dentro de esta categoría se incluyó al colibrí estrellita esmeraldeño (Chaetocercus berlepschi) y al tucán Dios te dé (Ramphastos ambiguus).

Debido al nivel de escolaridad de la mayoría de los participantes, la estrategia de filtro grueso-filtro fino se adaptó combinando niveles biológicos de biodiversidad (ecosistemas, comunidades y especies) y nivel de conservación (filtro grueso-filtro fino) para la explicación de conceptos y difusión de los resultados en la comunidad (ver figura 2). Asimismo, se realizó un ejemplo con el OCN más valorado por la comunidad a nivel de filtro grueso considerando la escala geográfica (ver figura 3 ).

En referencia a los cuatro objetos de conservación culturales, los escogidos fueron: 1) gastronomía, los pobladores describieron platos típicos como el seco de guanta, seco de venado y el ceviche de chumumo como los más populares en las comunidades; 2) fiestas patronales, siendo la fundación de la comuna, Virgen de las Mercedes y día de los difuntos como las más celebradas; $y, 3$ ) restos arqueológicos (huacas). El cuarto objeto siempre fue fluctuante entre la edificación (e.g. casa comunal), la memoria colectiva (e.g. historia de la comuna), la institución social (e.g. comuna) y el conocimiento y prácticas locales (e.g. elaboración de artesanías en tagua y paja toquilla) (figura 4); de estos, el último es casi inexistente dentro de la comunidad de Loma Alta.

\section{DISCUSIÓN}

La metodología de diseño para la conservación ha sido utilizada por entidades académicas, organizaciones gubernamentales y no gubernamentales en los EE. UU. y América Latina, como parte de la planificación ecorregional. Esta metodología ha sido idónea para este tipo de investigación; y el área, 


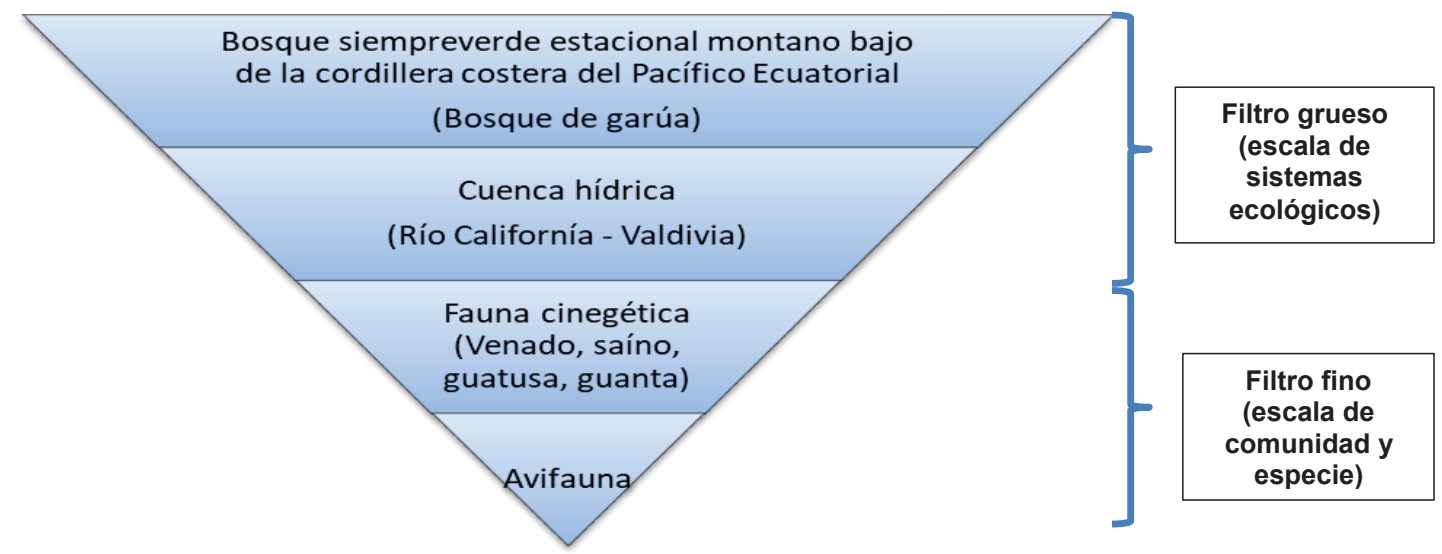

Figura 2. Objetos de conservación natural $(\mathrm{OCN})$ adaptados de la técnica filtro grueso-filtro fino.

Fuente: elaboración propia.

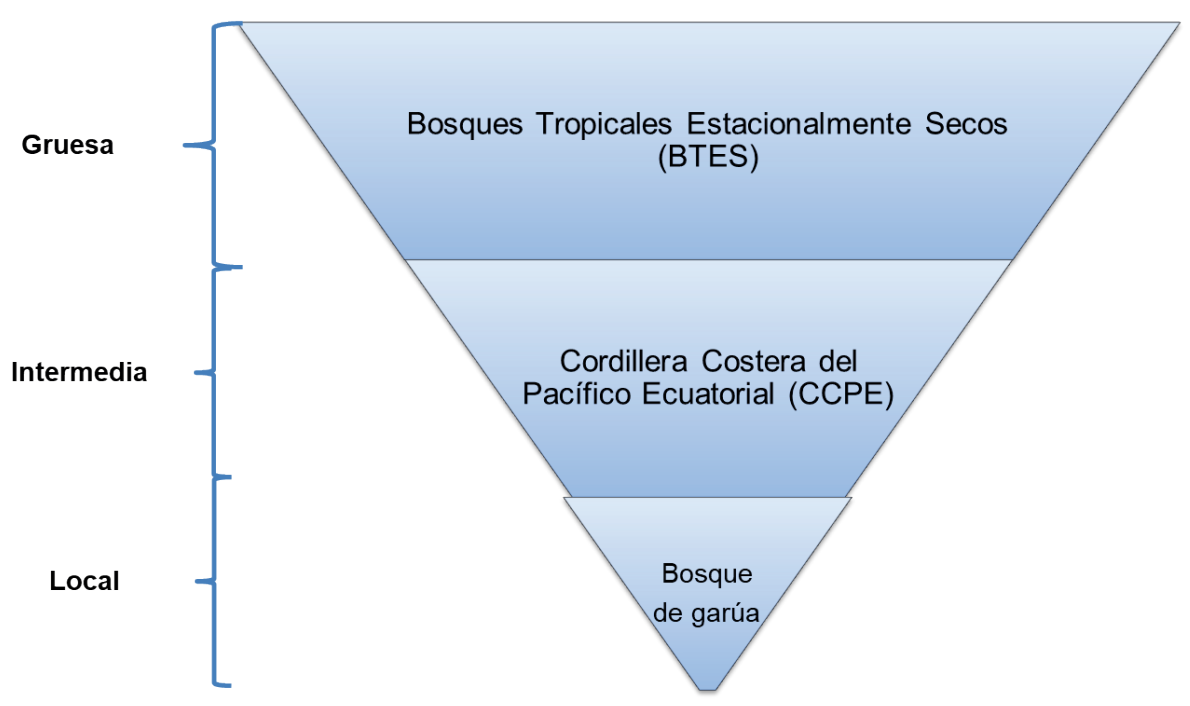

Figura 3. Objeto de conservación terrestre de filtro grueso.

Fuente: elaboración propia.

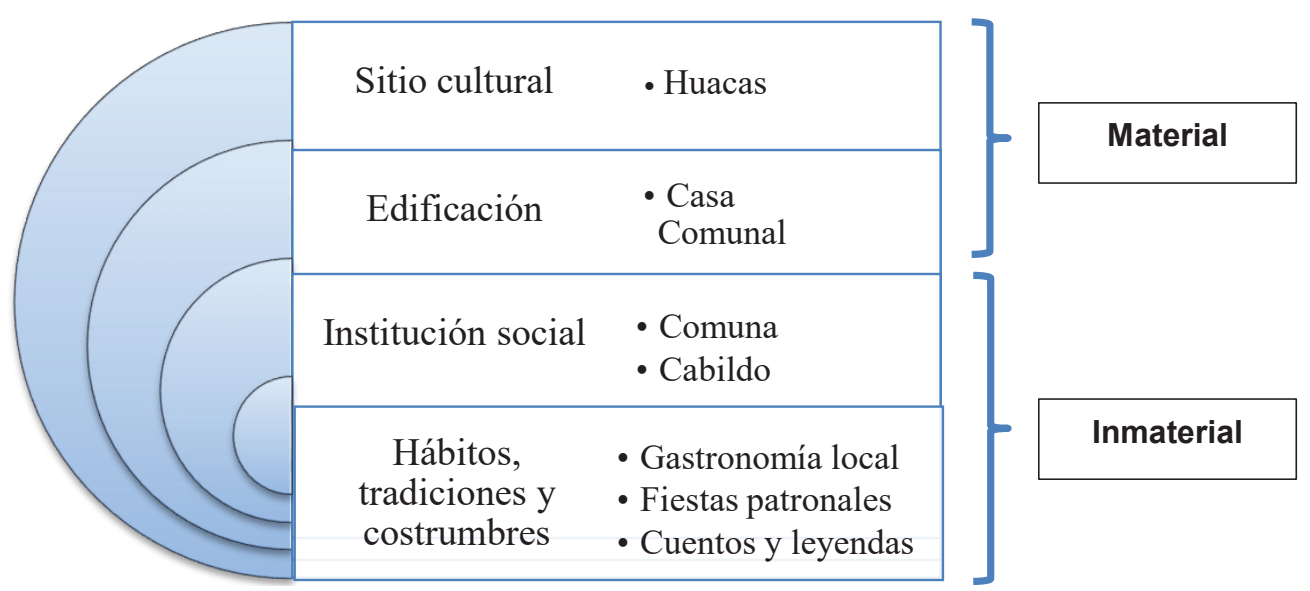

Figura 4. Objetos de conservación culturales.

Fuente: elaboración propia. 
como lo indican Chávez et al. (2014), es cuantitativa, pues emplea criterios ecológicos, biofísicos, sociales, económicos y políticos aplicados a grandes espacios geográficos y todo tipo de ecosistemas, por lo que además se considera que es sistemática, eficiente, repetible y participativa.

Asimismo, se debe recalcar que los resultados del FODA y las acciones propuestas son insumos para el desarrollo de una estrategia local que fortalezca la gestión territorial integral y que han sido obtenidos desde un conocimiento transdisciplinario, el cual refleja la dinámica en la gestión de recursos y sus organizaciones. Así, los resultados de la Planificación para la Conservación de Áreas (PCA) enfocados en los objetos de conservación naturales, provenientes de los comuneros, guardan relación con la lista preelaborada que se obtuvo con los expertos, pues ambas coinciden en que se debe conservar el bosque húmedo, a partir de los 400 $\mathrm{m}$ conocido como el bosque de garúa. La conservación de estos bosques es imperativa debido a que aportan con diversidad taxonómica y servicios ambientales para la sobrevivencia humana local en un área destacada por el clima seco y la vegetación xerofítica. Por ello, la conservación de estos bosques en la PSE debe ser una prioridad en la agenda nacional, provincial y local, debido a que en estas dos comunidades se protege la remanente flora, diversa, representativa y continua.

De esta manera, la conservación de la biodiversidad necesita información científica actualizada para el diseño de estrategias y políticas regionales de desarrollo, así como para la elaboración de planes de conservación y uso sostenible de la biodiversidad (Alvarado et al., 2011). La investigación científica descriptiva, según Zamora (2002), es un aporte básico y fundamental para gestionar la naturaleza; $y$, para hacerlo de una manera eficiente, se debe analizar también los procesos ecológicos y sus mecanismos con el fin de tomar mejores decisiones. Por lo tanto, los resultados de esta investigación permiten optimizar la gestión ambiental al conocer los valores a conservar presentes en los bosques protectores de la PSE, ya que las comunas carecen de la actualización de información biológica y de herramientas de planificación para una gestión eficiente.

La gestión sostenible, de acuerdo con Millennium Ecosystem Assessment (2005), necesita de un cambio sustancial en las instituciones y en la gobernanza, así como en las políticas para reducir la severidad de los problemas que enfrentan los ecosistemas. La democratización de la temática ambiental, a partir de la nueva Constitución de la
República del Ecuador, ha sido un cambio de paradigma en la conservación y gestión de la biodiversidad, la cual ha promovido una gestión ambiental eficiente y una gobernanza participativa.

En la PSE, la gobernanza participativa es un constituyente primordial en el manejo de los recursos debido a la identidad cultural indígena-campesina y la cosmovisión biocentrista y holística de las comunidades rurales. De allí que Loma Alta liderara los procesos de conservación en la $\mathrm{CCHC}$, que según Astudillo et al. (2015) se inició en 1987, protegiendo una superficie de 1858,25 ha de su bosque. Asimismo, la conservación mediante uso ha tenido experiencias exitosas en comunidades indígenas; un ejemplo de ello también aconteció en Loma Alta, donde Becker et al. (2005) demostraron el potencial de la participación social en los procesos de gobernanza, los cuales a través de la investigación científica a largo plazo lograron conservar la biodiversidad y establecer el anidamiento institucional local y nacional. Otros ejemplos de gobernanza participativa y de conservación mediante uso se presentan en la provincia de Napo, en la Reserva de Biosfera Sumaco (Torres et al., 2013), y en Honduras y México, en el bosque seco tropical mesoaméricano (Barrance et al., 2009).

Los resultados de Becker et al. (2005) coinciden con las investigaciones de Ostrom (2010) sobre la gobernanza de las comunas enfocada en el capital social y la acción colectiva en el uso del capital natural, así como la gobernanza policéntrica mediante el análisis institucional multinivel. A partir de estas investigaciones previas, se continuó con los procesos de gobernabilidad participativa y de fortalecimiento del anidamiento institucional y multinivel en el desarrollo de este proyecto que se refleja en los resultados, donde se vinculó a la academia (por ejemplo, Universidad de Especialidades Espirítu Santo), a autoridades locales (por ejemplo, cabildos de Loma Alta y Dos Mangas, M. I. Municipalidad de Santa Elena) y autoridades nacionales (por ejemplo, Ministerio del Ambiente y Ministerio de Turismo).

Pese al avance en la conservación de sus recursos y del incentivo económico anual que las comunas reciben del programa Socio Bosque, existe la necesidad de integrar estas áreas protegidas al desarrollo regional y local, donde la perspectiva de conservación de la biodiversidad se integre a la planificación ecorregional y a la gestión del territorio. Una estrategia de planificación y conservación que ha sido impulsada desde las dos últimas décadas del siglo pasado ha sido la conectividad ecológica territorial, la cual mantiene una conexión funcional 
derivada de las habilidades de movimiento de las especies para conservar flujos ecológicos a escala de paisaje (EUROPARC-España, 2009).

Un enfoque de la conectividad ecológica son los corredores de conservación como estrategias de planificación. Los corredores de conservación son instrumentos de manejo que acoplan las necesidades de desarrollo humano con las prioridades de conservación, donde su función es permitir la conexión entre áreas protegidas o fragmentos de hábitats para mantener la sostenibilidad de los procesos ecológicos naturales y aumentar la movilidad e intercambio genético entre especies (Critical Ecosystem Partnership Fund, 2005). Dentro de la ecorregión terrestre del Chocó-Darien-Ecuador occidental se creó el Corredor de Conservación Chocó-Manabí, el cual posee una estrategia de conservación y establece alianzas estratégicas entre Ecuador-Colombia y EE. UU.

A pesar de esta iniciativa, la PSE y sus áreas protegidas no se incluyeron en el desarrollo de esta estrategia. Los resultados de Astudillo et al. (2019a, b) acerca del análisis de ordenación espacial de las formaciones vegetales y similitud florística en varias localidades de la $\mathrm{CCHC}$ resaltan la afinidad vegetal ligado a un gradiente altitudinal y no por área, lo cual refuerza el concepto de corredor ecológico. Por otra parte, los resultados de esta investigación destacan el esfuerzo de los actores sociales locales y nacionales para impulsar proyectos que fortalezcan sus capacidades de planificación y manejo de sus recursos naturales y culturales. A nivel de planificación turística, Chóez et al. (2017) plantearon una propuesta de sendero escénico y de aventura que recorra las dos comunidades para impulsar la sostenibilidad ambiental y humana; sin embargo, esta iniciativa no ha prosperado debido a la falta de organización de ambas comunidades, falencias en el manejo de sus recursos y un déficit en la planificación estratégica a largo plazo.

\section{CONCLUSIONES}

1. La gestión eficiente de los bosques tropicales estacionalmente secos ubicados en la PSE presenta el reto de fortalecer las capacidades de planificación y manejo ambiental local.

2. El manejo de los recursos naturales y culturales en Santa Elena enfrenta falencias en el ámbito técnico-administrativo por la carencia de un plan de manejo y un departamento administrativo autónomo que dirijan los procesos socioambientales en las áreas protegidas y fomente el desarrollo sustentable mediante la diversificación de mecanismos financieros innovadores basados en los servicios ambientales.

3. El reconocimiento del bosque y la institucionalidad social del cabildo como objetos de conservación natural y cultural afianzan el concepto de conservación mediante el uso de los recursos que funciona a nivel comunal para Santa Elena, pero debe de ser compatible con las condiciones locales de cultura, tenencia de tierra y las necesidades de desarrollo local.

4. La gobernanza ambiental actual en la PSE debe reforzar métodos de gestión participativa, inclusivos, informados y justos para alcanzar una sostenibilidad de las áreas protegidas.

\section{AGRADECIMIENTOS}

Al Centro de Investigaciones de la Universidad de Especialidades Espíritu Santo (UEES) por el financiamiento para la ejecución de este proyecto (UEES-2014-ART-002) y a los decanos, docentes y estudiantes de las facultades de Artes Liberales y Ciencias de la Educación (escuelas de Ciencias Ambientales, Psicología y Educación), Economía (Escuela de Turismo) y Comunicación (Escuela de Diseño y Comunicación). También, al Ministerio del Ambiente del Ecuador (MAE), a través de su Dirección provincial de Santa Elena, por el permiso de investigación correspondiente N. ${ }^{\circ}$ 017-15 IC-FAUDPSE-MA y al M. I. Municipalidad de Santa Elena, Lic. Dionicio Gonzabay, alcalde de Santa Elena. A las autoridades locales, Abg. Gustavo de la A (presidente de Loma Alta) y Sr. Ángel Merchán (presidente de Dos Mangas) y los guardaparques que aportaron en la realización de este proyecto.

\section{REFERENCIAS BIBLIOGRÁFICAS}

[1] Acción en el Biocorredor (2012). Plan de acción del Biocorredor territorio Chongón Colonche: biocorredores para el buen vivir. Guayaquil, Ecuador: FIDES, SGP, GEF y UNDP.

[2] Aguirre, N. yErazo,A. (2017). Valoración económica ambiental del compartimiento leñoso como una alternativa para conservar la biodiversidad del bosque seco de la provincia de Loja, Ecuador. Bosques Latitud Cero, 7(1), 89-107.

[3] Altmann, P. (2013). El Sumak Kawsay en el discurso del movimiento indígena ecuatoriano. Indiana, 30, 283-299. Recuperado de https://www.iai.spk-berlin.de/fileadmin/ dokumentenbibliothek/Indiana/Indiana_30/ IND_30_2013_283-299_Altmann.pdf. 
[4] Alvarado, J.; Herrera, B.; Corrales, L.; Asch, J. y Paaby, P. (2011). Identificación de las prioridades de conservación de la biodiversidad marina y costera en Costa Rica. Revista de Biología Tropical, 59(2), 829-842. Recuperado de https://revistas.ucr.ac.cr/index.php/rbt/ article/view/3143/3050.

[5] Astudillo, E. (2010). El desarrollo sostenible comunitario en un ENP de alto interés científico: el caso de la comuna Loma Alta y su reserva ecológica. (Tesis de maestría). Universidad Autónoma de Madrid, Madrid.

[6] Astudillo-Sánchez, E., Pérez, J., Troccoli, L., Aponte, H., \& Tinoco, O. (2019b). Flora Leñosa del Bosque de Garúa de la Cordillera Chongón Colonche, Santa Elena-Ecuador. Ecología Aplicada, 18(2), 155-169. http://dx.doi. org/10.21704/rea.v18i2.1334

[7] Astudillo, E.; Pérez, J. y Fabara, M. (2015). Árboles y arbustos: una relación con la avifauna. Samborondón, Ecuador: Universidad Espíritu Santo.

[8] Astudillo-Sánchez, E., Pérez, J., Troccoli, L., \& Aponte, H. (2019a). Composición, estructura y diversidad vegetal de la Reserva Ecológica Comunal Loma Alta, Santa Elena, Ecuador. Revista Mexicana de Biodiversidad, 90(1), 1-25. https://doi.org/10.22201/ ib.20078706e.2019.90.2871

[9] Barrance, A.; Schreckenberg, K. y Gordon, J. (2009). Conservación mediante el uso: lecciones aprendidas en el bosque seco tropical mesoamericano. Londres, Inglaterra: Overseas Development Institute.

[10] Becker, D. (1999). Protecting a garúa forest in Ecuador: the role of institutions and ecosystem valuation. Ambio, 28(2), 156-161. Recuperado de https://lifenetnature.org/download/docs/ lifenetnature-org__protecting-garua-forest.pdf.

[11] Becker, D.; Agreda, A.; Astudillo, E.; Costantino, M. y Torres, P. (2005). Community-based monitoring of fog capture and biodiversity at Loma Alta, Ecuador: enhanced social capital and institutional cooperation. Biodiversity and Conservation, 14, 2695-2707. Recuperado de http://www. monitoringmatters.org/articles/full9.pdf.

[12] Best, B. y Kessler, M. (1995). Biodiversity and conservation in tumbesian Ecuador and Peru. Cambridge, Inglaterra: BirdLife International. Recuperado de https://pavaaliblanca.weebly. com/uploads/5/0/3/0/50301069/best_kessler. pdf.
[13] Chávez, H.; González, M. y Hernández, P. (2014). Metodologías para identificar áreas prioritarias para conservación de ecosistemas naturales. Revista Mexicana de Ciencias Forestales, 6(27), 8-23. Recuperado de http://www.scielo.org.mx/pdf/remcf/v6n27/ v6n27a2.pdf.

[14] Chóez, M.; Dávila, C. y Astudillo, E. (2017). Diagnóstico del potencial emprendedor $y$ turístico en la comuna Loma Alta, Santa Elena. Samborondón, Ecuador: Universidad Espíritu Santo.

[15] Cifuentes, M.; Izurieta, A. y Henrique, $H$. (2000). Medición de la efectividad del manejo de áreas protegidas. Turrialba, Costa Rica: World Wildlife Fund.

[16] Columba, K. (2013). Manual para la gestión operativa de las áreas protegidas del Ecuador. Quito, Ecuador: Ministerio del Medio Ambiente / Punto Verde.

[17] Critical Ecosystem Partnership Fund (2005). Perfil del ecosistema: Corredor de Conservación Chocó-Manabí, ecorregión terrestre prioritaria del Corredor Chocó-DarienEcuador occidental (hotspot). Colombia $y$ Ecuador. Recuperado de https://www.cepf.net/ sites/default/files/final.spanish.choco-darienwestern-ecuador.choco_.ep_.pdf.

[18] EUROPARC-España (2009). Conectividad ecológica y áreas protegidas. Herramientas y casos prácticos. Madrid, España: FUNGOBE. http://www.redeuroparc.org/system/files/ shared/monografia2.pdf.

[19] Gobierno autónomo descentralizado municipal Santa Elena (2014). Plan de desarrollo y ordenamiento territorial-Cantón Santa Elena 2014-2019. Recuperado de http://app.sni. gob.ec/sni-link/sni/PORTAL_SNI/data_sigad_ plus/sigadplusdiagnostico/0960001540001 PLAN\%20DE\%20DESARROLLO\%20Y\%20 ORDENAMIENTO\%20TERRITORIAL\%203001-2015-2\%20fin_19-02-2015_09-41-20.pdf.

[20] Granizo, T.; Molina, M.; Secaira, E.; Herrera, B.; Benítez, S.; Maldonado, O.; Libby, M.; Arroyo, P.; Ísola, S. y Castro, M. (2006). Manual de planificación para la conservación de áreas, PCA. Quito, Ecuador: The Nature Conservancy y The United States Agency for International Development.

[21] Groves, C.; Valutis, L.; Vosick, D.; Neely, B.; Wheaton, K.; Touval, J. y Runnels, B. (2000). Diseño de una geografía de la esperanza. 
Manual para la planificación de la conservación ecorregional (vols. 1 y 2). Arlington, EE. UU.: The Nature Conservancy.

[22] Gurrutxaga, M. y Lozano, P. (2009). La integración de la conectividad ecológica en los instrumentos de ordenación y planificación territorial: una revisión. Boletín de la Asociación de Geógrafos Españoles, (49), 45-66.

[23] López, E. y Peralta, P. (2016). Breve síntesis histórica y reflexiones acerca de la continuidad étnica, sentido de etnicidad e identidad cultural en la región de Santa Elena, Ecuador. Revista Científica y Tecnológica UPSE, 3(3), 99-105.

[24] Millennium Ecosystem Assessment (2005). Ecosystems and human well-being. Synthesis. Washington, USA: Island Press.

[25] Ministerio del Ambiente del Ecuador (2012a). Línea base de deforestación del Ecuador continental. Quito, Ecuador: Ministerio del Ambiente del Ecuador / Socio Bosque.

[26] Ministerio del Ambiente del Ecuador (2012b). Sistema de clasificación de los ecosistemas del Ecuador continental. Quito, Ecuador: Subsecretaría de Patrimonio Natural.

[27] Ministerio del Ambiente del Ecuador (2017). Análisis de vulnerabilidad local al cambio climático del sector ganadero en las zonas de implementación del proyecto $\mathrm{MGCl}$ en la provincia de Santa Elena. Quito, Ecuador: Ministerio de Agricultura y Ganadería.

[28] Organización de las Naciones Unidas para la Alimentación (2016). El estado de los bosques del mundo 2016. Los bosques y la agricultura: desafíos y oportunidades en relación con el uso de la tierra. Roma, Italia: Food and Agriculture Organization of the United Nations (FAO). Recuperado de http://www.fao.org/3/i5588s/ i5588s.pdf.

[29] Ostrom, E. (2010). Polycentric systems for coping with collective action and global environmental change. Global Environmental Change, 20(4), 550-557.

[30] Ponce, H. (2006). La matriz FODA: una alternativa para realizar diagnósticos y determinar estrategias de intervención en las organizaciones productivas y sociales. Contribuciones a la Economía, 2, 1-16.

[31] Thompson, A. A.; Strickland, A. J.; Colado, P. I. y Carreón, M. A. S. (1998). Dirección y administración estratégicas: conceptos, casos y lecturas. México, D. F., México: McGraw-Hill.

[32] Torres, B.; Starnfeld, F.; Vargas, J.; Ramm, G.; Chapalbay, R.; Rios, M.; Gómez, A.; Torricelli, Y.; Jurrius, I.; Tapia, A.; Shiguango, J.; Torres, A. Velasco, C.; Murgueytio, A. y CordobaBahle, D. (2013). Gobernanza participativa en la Amazonía del Ecuador: recursos naturales y desarrollo sostenible. Puyo, Ecuador: Universidad Estatal Amazónica, Ministerio del Ambiente del Ecuador, Gobierno Autónomo Descentralizado Provincial de Napo y Cooperación Alemana al Desarrollo.

[33] Zamora, R. (2002). Los espacios protegidos necesitan una gestión activa. Quercus, 191, 64-65. 


\title{
Management of the tropical dry forests in Santa Elena Province: a conservation approach
}

\author{
EvelYNG Astudillo-SÁNCHEZ \\ JAME PÉREZ FLOR ${ }^{2}$ \\ Gilmer Medina ${ }^{3}$ \\ Ana Medina ${ }^{4}$
}

\begin{abstract}
Seasonally dry tropical forests are recognized for their biological and economic importance, as they possess a high number of endemic species and provide various ecosystem functions. However, the lack of a biodiversity conservation plan threatens comprehensive environmental management in the protected areas of Santa Elena Province, Ecuador. For this reason, a diagnosis was made in which conservation targets were identified to strengthen local environmental management capacity. Furthermore, Conservation Action Planning (CAP) and Strengths, Weaknesses, Opportunities and Threats (SWOT) methodologies were used to identify conservation priorities and resource management constraints. The analysis revealed technical-administrative flaws, and because of this an efficient approach to environmental management was sought. In addition, the garúa forest was recognized as a natural conservation target and the cabildo (community council) was recognized as a cultural conservation target. Participatory environmental governance needs to be strengthened to improve the management of protected areas.
\end{abstract}

Keywords: Protected areas; seasonally dry tropical forest; local environmental management; Santa Elena (Ecuador).

\section{INTRODUCTION}

The creation of protected areas is a legal tool for the conservation of nature. The establishment and management of protected areas is the most widely used strategy for the conservation of ecosystems and their biodiversity in the world (Chávez et al., 2014). However, this global strategy needs to be accompanied by planning and integrated into a matrix with socio-economic and political aspects that require multidisciplinary work (Gurrutxaga \& Lozano, 2009). Protected areas are not exempt from conservation problems due to human presence, either within or on the periphery of the area. Deforestation as a problem is a challenge in the management of protected areas and is one of the main sources of $\mathrm{CO}_{2}$ emissions contributing to climate change (CC), in addition to being the second largest threat that biodiversity faces.

Deforestation causes an ecosystem to be fragmented into patches that lack diversity of species due to the edge effect, affecting trophic interaction networks (EUROPARC-España, 2009). The Food and Agriculture Organization of the United Nations (2016) registered for the tropical region, in the period of 2000-2010, a loss of forest cover of 7 million hectares and a net annual increase in agricultural area of 6 million hectares.

In Ecuador, the National System of Protected Areas (SNAP, by its acronym in Spanish) was established in 1959 with the creation of the Galapagos National Park; later, in 1996, the Ministry of the Environment was constituted as the environmental authority and sole administrator of SNAP (Columba, 2013). In 2008, environmental policy in the country evolved towards a biocentric approach with the new constitution and sumak kawsay-an indigenous worldview-, which recognizes the plurinationali-

1 Biologist from the Universidad de Guayaquil, Ecuador. Master in Protected Natural Spaces from the Universidad Autónoma de Madrid, Spain. Associate researcher at the Research Center, Universidad Espíritu Santo, Samborondón, Ecuador. ORCID: 0000-0003-0006-2730

E-mail: eveavesecuador@gmail.com

2 Biologist from the Universidad de Guayaquil, Ecuador. Currently Director of Jardín Botánico de Guayaquil and independent consultant.

E-mail:.jperez_40@hotmail.com

3 Geographer from the Universidad Nacional Mayor de San Marcos and Master in Environmental Science from the Universidad Nacional Mayor de San Marcos. Currently working as Professor at the School of Industrial Engineering of the Universidad Privada del Norte. Dean of Colegio de Geografos del Peru. Lima, Peru. E-mail: gilmer.medina@upn.edu.pe

4 Chemical Engineer from the Universidad Nacional Mayor de San Marcos. Professor at the School of Industrial Engineering of the Universidad Nacional Mayor de San Marcos, Lima, Peru. E-mail: amedinae@unmsm.edu.pe 
ty and interculturality of the State, grants rights to the environment and confers territorial autonomy (Altmann, 2013). In this way, society was involved in the management of protected areas and SNAP was structured into four subsystems: 1) State Natural Heritage Areas [Patrimonio de Áreas Naturales del Estado, PANE], 2) Decentralized-Autonomous Governments [Gobiernos Autónomos Descentralizados, GAD], 3) indigenous and Afro-Ecuadorian community subsystems and 4) private subsystems (Columba, 2013).

Santa Elena Province protects $28 \%$ of its land area, while $4 \%$ is part of the community subsystem (Ministerio del Ambiente del Ecuador, 2017). Despite this, it has been the province, along with Guayas, most affected by deforestation during the period of 2000-2008 (Ministerio del Ambiente del Ecuador, 2012a). Deforestation has been reported since the 1990 s by authors such as Dodson and Gentry, Parker and Carr, Bonifaz and Cornejo, Astudillo, Pérez and Fabara, among others, who confirm that the area has been disturbed by selective extraction, while in some places the forest has been converted into grazing areas for livestock.

The ecosystems in Santa Elena Province (PSE) are part of the seasonally dry tropical forests (SDTF) of the Equatorial Pacific, which form a floristic region with high diversity and endemism; however, it is considered one of the most threatened tropical ecosystems due to the level of disturbance and fragmentation (Best \& Kessler, 1995).

The economic contribution of the dry forests is known, but not quantified, as research is lacking, particularly in the PSE, as the few investigations have occurred specifically in Southern Ecuador. In addition, dry forests have been underestimated, according to Aguirre and Erazo (2017), who emphasize the importance of research in ecological and financial valuation of these forests, as they provide a diversity of ecosystem services. An example of this is the study in the community of Loma Alta conducted by Becker (1999), which focuses on the hypothesis that the community members do not seem to appreciate the indirect values of the upland forest, which provides them with the water supply with which they subsist and use for irrigation in agriculture.

The interdependence between human well-being and provision of forest ecosystem services is increasing; accordingly, the functioning of forests needs to be ensured in the long term, where protected areas play an essential role in the conservation of natural and cultural resources. Planning and efficient management of these resources are a challenge for the managers of protected areas, who are bound by a series of legal, administrative, social and financial constraints, among others (Cifuentes et al., 2000).

As a first step, it is essential to have a planning strategy for the conservation of the Equatorial $\mathrm{Pa}$ cific SDTF located in the Chongón-Colonche Range (CCR) and to comprehend their natural and cultural values in order to preserve them. Therefore, the objective of this research is to identify the conservation targets present in two protected areas located in Santa Elena Province for the development of a local development strategy and to strengthen environmental management competency.

\section{METHODOLOGY}

\section{Study area}

Geopolitical aspects. The PSE has an area of 3 $762.8 \mathrm{~km}^{2}$ and is geopolitically divided into three cantons: Santa Elena (3 $\left.668.9 \mathrm{~km}^{2}\right)$, Salinas $(68.7$ $\mathrm{km}^{2}$ ) and La Libertad (25.3 $\left.\mathrm{km}^{2}\right)$ (see Figure 1). Santa Elena is the canton with the highest level of agricultural activity, where $14 \%$ of its area is dedicated to crops and $6 \%$ to pasture. This canton also has the largest forest resources, as in 2008 it reported an area of $31 \%$ native forest and $42 \%$ shrubby vegetation, evincing remarkable land use for conservation (Gobierno autónomo descentralizado municipal Santa Elena, 2014). In the canton of Santa Elena, two localities with protected areas were chosen: Loma Alta community $\left(01^{\circ} 52\right.$ 'S, $80^{\circ}$ $38^{\prime} \mathrm{W}$ ) (see Figure 1), which has a bosque protector (forest preserve) of 3218.19 ha known as Reserva Ecológica Comunal Loma Alta (ecological reserve); and, Dos Mangas community ( $\left.02^{\circ} 07^{\prime} \mathrm{S}, 80^{\circ} 18^{\prime} \mathrm{W}\right)$ (see Figure 1), which is part of the bosque protector Chongón-Colonche with an area of 83731 ha (Acción en el Biocorredor, 2012).

Biogeographical aspects. The Chongón-Colonche Range crosses the PSE at its northeastern end (see Figure 1). It has a variable climate influenced by sea currents and the altitudinal gradient. From June to November, the cold Humboldt Current causes a fog and drizzle effect known as garúa, which provides $40 \%$ of the water that the forest captures at 400 masl (Becker, 1999). The PSE has two well-defined seasons: a rainy season, from December to April, and a dry season, from June to October, which coincides with the garúa months. The annual average temperature in the Santa Elena canton is $23.4{ }^{\circ} \mathrm{C}$ and its annual average precipitation is $66 \mathrm{~mm} / \mathrm{year}$, with a variability of up to $200 \mathrm{~mm} / \mathrm{month}$ in the rainy season and close to 

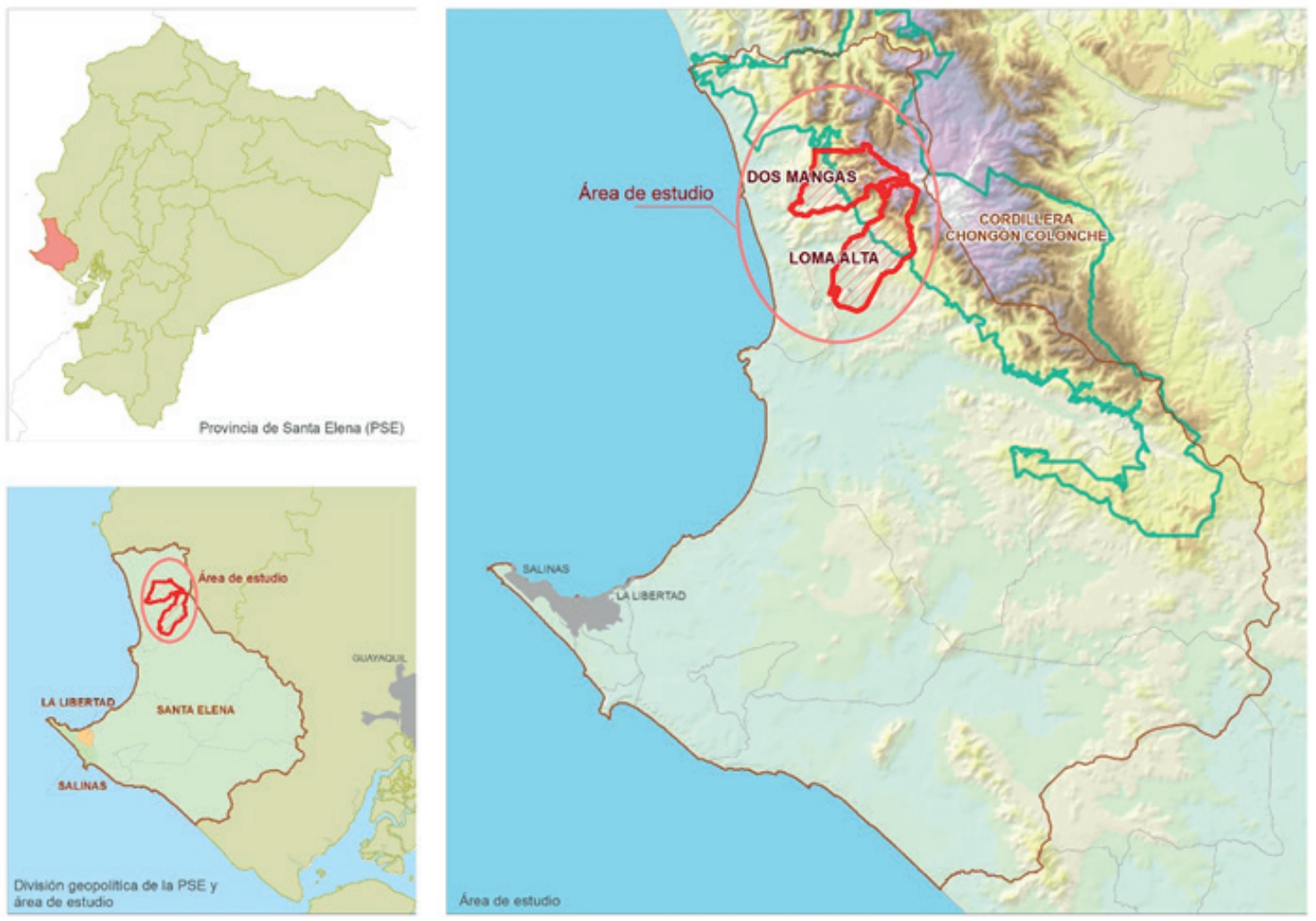

Figure 1. Geographical location of the Loma Alta and Dos Mangas communities, PSE.

Source: Prepared by the authors.

zero in the dry season (Gobierno autónomo descentralizado municipal Santa Elena, 2014).

Two biogeographical regions converge in the Chongón-Colonche Range: Chocó and Tumbes. Currently, they are merged and formally recognized as the Chocó-Darién-Western Ecuador Terrestrial Ecoregion, which extends from "the southeastern portion of Panamá, along the western portions of Colombia and Ecuador, as far as northwestern Perú" (Critical Ecosystem Partnership Fund, 2005, p. 6). This terrestrial ecoregion is known as the Tumbes-Chocó-Magdalena Hotspot, which is characterized by a diversity of ecosystems, from humid premontane forests to dry thorny scrub.

Floristically, the Chongón-Colonche Range (CCR) forms part of the Equatorial Pacific Coastal Range (EPCR), which includes the highlands and foothills of the Chongón-Colonche Range with a semi-arid to sub-humid bioclimate, where vegetation is typical of humid ecosystems starting at 400 masl and dry in lower areas (Ministerio del Ambiente del Ecuador, 2012b). Four ecosystems were identified in the Equatorial Pacific Coastal Range: 1) EPCR deciduous forest, located in the foothills of the CCR, on a bioclimatic floor of $>200$ masl, with an xeric-dry climate and tree stratum between 15 and $20 \mathrm{~m}$ high with deciduous species; 2) EPCR semi-deciduous forest, located on the ridges and slopes, on a bioclimatic floor >200 masl, with seasonal rainy sub-humid climate and tree stratum between 12 and 25 $\mathrm{m}$ high with $25 \%$ deciduous species; 3 ) EPCR seasonal premontane evergreen forest, located in a bioclimatic floor at elevation between 200-400 masl, with seasonal rainy sub-humid climate and average tree stratum of $20 \mathrm{~m}$ with emerging trees up to $30 \mathrm{~m}$ high; 4) EPCR seasonal montane evergreen forest, located on a bioclimatic floor at elevations between 400-860 masl, with seasonal rainy sub-humid climate and a multilayered forest of trees up to $25 \mathrm{~m}$ high (Ministerio del Ambiente del Ecuador, 2012b).

Socio-economic aspects. The inhabitants have a cultural identity typical of pre-Columbian peoples, such as Valdivia (4200-1500 BC), Manteño-Guancavilca and Milagro-Quevedo (Integration Period, 800 B.C. - A.D. 1535), which were characterized by the ethnic socio-cultural dynamics established by complex societies (López \& Peralta, 2016). According to the Ley de Comunas enacted in 1936: "Ios descendientes de estos pueblos precolombinos han conservado cierta estructura social y económica, congregándose en comunidades indígenas y 
campesinas [...], la cual garantiza el ejercicio de los derechos colectivos indígenas o afroecuatorianos que se autodefinen con nacionalidades de raíces ancestrales" [the descendants of these pre-Columbian peoples have preserved a certain social and economic structure, gathering in indigenous and rural communities [...], which guarantees the exercise of indigenous or Afro-Ecuadorian collective rights who self-identify with ancestral nationalities] (Astudillo, 2010, p. 22).

\section{METHODOLOGY}

The chosen planning methodology was the conservation design model created by The Nature Conservancy (TNC), which has four components: 1) ecoregional conservation planning, where areas known as conservation portfolios are selected; 2) planning for site conservation or strategy development, either in individual sites or in a network of protected areas; 3 ) conservation action or decision-making, where the type of support is defined; and 4) success measurement, where actions are evaluated (Granizo et al., 2006).

In accordance to the proposed objective, this research focuses on the second component of the conservation design model; although it should be noted that some of the results of the first component are presented here during the selection of the study area, as they are part of the conservation portfolio for the PSE.

It was necessary to know the conservation priorities within each locality to develop strategies; therefore, the Conservation Action Planning (CAP) methodology was as a tool, which includes a diagnostic phase of the area, the first step of which is the selection of conservation targets (Granizo et al., 2006). To complement the diagnosis, the Strengths, Weaknesses, Opportunities and Threats (SWOT) analysis technique was used to determine the potential and limitations of the management and public use of the natural and cultural resources of the communities and their protected areas. SWOT analysis is a strategic planning tool, presented in a matrix, that assesses the strong and weak factors within an organization or project, as well as the opportunities and threats of its external environment (Ponce, 2006).

The SWOT analysis and the selection of conservation targets were carried out in four participatory workshops in the years 2015, 2016 and 2017, with approximately twenty participants for each workshop; local experts, social actors and representatives of governmental and non-governmental environmental organizations were also involved.

Conservation targets are unique elements or characteristics found at different levels of biological organization and geographical scales that enable the identification of conservation sites for ecoregions or threats and develop strategies for an area (Groves et al., 2000). Given the objective of the project and logistics in the areas, the first workshop was conducted with a multi-disciplinary group of experts in the area of engineering (environmental, forest, civil, mechanical, and tourism engineers) and biologists, who helped with the initial goal of designing the conservation portfolio for the PSE. The workshops were held in situ with the communities, and via brainstorming, natural conservation targets (NCT) and cultural conservation targets (CCT) were catalogued.

According to the categories and requirements described by Granizo et al. (2006), a total of eight elements were chosen in each locality, four for each type of target, and the coarse/fine filter approach was used for the definition of targets. This technique consists of an inverted pyramid, which specifies four spatial scales (regional, coarse, intermediate and local) and describes three levels of biological organization (species, communities and ecosystems) (Groves et al., 2000). According to Granizo et al. (2006), the coarse filter protects strategic communities and ecosystems that ensure their functionality and viability; whereas, the fine filter preserves species that slip through the coarse filter or are not protected.

\section{RESULTS}

The SWOT analysis was organized in thematic areas: local economic development; social development and human capital; institutional development and citizen participation; territorial planning; and infrastructure development. See the table 1.

Based on the diagnosis and SWOT analysis, the following information relevant to the development of a local strategy is presented; several necessary measures to preserve resources, ensure ecological integrity and strengthen local environmental management capacity are proposed.

At the technical level, the development of a management plan for each protected area is imperative, because it will contain zoning proposal, where the various stakeholders can reach the mutual compatibility of their economic interests in special functional areas, and conservation areas. It is suggested that the regulations governing the use of natural 
Table 1. SWOT matrix of Loma Alta and Dos Mangas communities and their protected areas.

\begin{tabular}{|c|c|c|c|}
\hline Strengths & Opportunities & Weaknesses & Threats \\
\hline $\begin{array}{l}\text { Presence of natural and cultural } \\
\text { resources in the communities }\end{array}$ & $\begin{array}{l}\text { Potential for scientific research } \\
\text { in various areas }\end{array}$ & Cabildo functions overload & Missing opportunities \\
\hline $\begin{array}{l}\text { Provision of ecosystem services } \\
\text { by protected areas }\end{array}$ & $\begin{array}{c}\text { Potential to implement various } \\
\text { innovative financial mechanisms } \\
\text { for biodiversity conservation such } \\
\text { as Payments for Environmental } \\
\text { Services (PES) }\end{array}$ & $\begin{array}{c}\text { Community's lack of experience, } \\
\text { motivation and training to initiate } \\
\text { projects and develop activities } \\
\text { in the economic, tourism and } \\
\text { environmental sectors }\end{array}$ & $\begin{array}{c}\text { Current economic crisis facing } \\
\text { the country to support established } \\
\text { programs }\end{array}$ \\
\hline $\begin{array}{c}\text { High levels of biodiversity and } \\
\text { endemism }\end{array}$ & $\begin{array}{c}\text { Support from the Ministry of } \\
\text { the Environment (MAE) for the } \\
\text { protected area to be annexed to } \\
\text { the PANE }\end{array}$ & $\begin{array}{l}\text { Lack of continuous technical } \\
\text { advice on planning, community } \\
\text { management and development } \\
\text { issues }\end{array}$ & \multirow{2}{*}{$\begin{array}{c}\text { Increasing environmental } \\
\text { problems such as climate } \\
\text { change, loss of vegetation cover, } \\
\text { watershed degradation, habitat } \\
\text { fragmentation and pollution of } \\
\text { natural resources }\end{array}$} \\
\hline $\begin{array}{c}\text { Possession of legal docu- } \\
\text { mentation declaring the area } \\
\text { as a bosque protector (forest } \\
\text { preserve) }\end{array}$ & $\begin{array}{l}\text { Potential to create an environ- } \\
\text { mental brand with products } \\
\text { derived from biodiversity }\end{array}$ & $\begin{array}{l}\text { Community's lack of } \\
\text { self-management }\end{array}$ & \\
\hline $\begin{array}{l}\text { Membership in the Socio } \\
\text { Bosque program and annual } \\
\text { receipt of a financial incentive } \\
\text { for resource management }\end{array}$ & $\begin{array}{l}\text { Support from the municipal and } \\
\text { provincial GAD }\end{array}$ & $\begin{array}{l}\text { Very limited financial resources } \\
\text { for conservation programs, } \\
\text { despite receiving an economic } \\
\text { incentive from Socio Bosque for } \\
\text { this purpose }\end{array}$ & $\begin{array}{l}\text { Almost exclusive economic de- } \\
\text { pendence on the Socio Bosque } \\
\text { program to manage natural } \\
\text { resources }\end{array}$ \\
\hline $\begin{array}{l}\text { Declaration of Important Bird } \\
\text { Area (IBA) in both communities }\end{array}$ & $\begin{array}{l}\text { Potential for sustainable } \\
\text { community tourism initiatives in } \\
\text { various forms }\end{array}$ & $\begin{array}{l}\text { Limited knowledge of English } \\
\text { necessary to meet the demand } \\
\text { of international tourism }\end{array}$ & $\begin{array}{l}\text { Insufficient national recognition, } \\
\text { appreciation and promotion of } \\
\text { the protected area }\end{array}$ \\
\hline $\begin{array}{l}\text { Interest on the part of the cabil- } \\
\text { do, park rangers and other key } \\
\text { actors in improving the planning } \\
\text { and management of their natural } \\
\text { and cultural resources }\end{array}$ & $\begin{array}{l}\text { Supported by national and inter- } \\
\text { national environmental NGOs }\end{array}$ & $\begin{array}{l}\text { Lack of an administrative struc- } \\
\text { ture and strategic leadership to } \\
\text { manage the protected area }\end{array}$ & $\begin{array}{l}\text { Limited organizational culture and } \\
\text { leadership to initiate economic } \\
\text { and social ventures }\end{array}$ \\
\hline $\begin{array}{c}\text { Increasing interest of ecotourism } \\
\text { in protected forest areas in the } \\
\text { PES }\end{array}$ & $\begin{array}{l}\text { Increasing recognition of the } \\
\text { protected area at international } \\
\text { level owing to research and } \\
\text { dissemination efforts }\end{array}$ & \multirow{2}{*}{$\begin{array}{c}\text { Loma Alta does not have a team } \\
\text { of community environmental } \\
\text { interpreters to pursue guided } \\
\text { tourist activities }\end{array}$} & $\begin{array}{c}\text { Geographical location of Loma } \\
\text { Alta and difficult access to the } \\
\text { protected area }\end{array}$ \\
\hline $\begin{array}{c}\text { Predisposition of young } \\
\text { community members to train } \\
\text { for and develop sustainable } \\
\text { tourism activities }\end{array}$ & $\begin{array}{l}\text { Possibility of establishing strategic } \\
\text { alliances with universities }\end{array}$ & & $\begin{array}{l}\text { Competition in the supply of } \\
\text { tourist products in the PSE }\end{array}$ \\
\hline High level of public safety & $\begin{array}{l}\text { Establishment of (e.g. Christmas } \\
\text { bird count) and cultural (sabores } \\
\text { y saberes) events established }\end{array}$ & \multirow{2}{*}{$\begin{array}{l}\text { Low level of higher education } \\
\text { and lack of knowledge on the } \\
\text { part of community leaders for } \\
\text { the management of the protec- } \\
\text { ted area }\end{array}$} & $\begin{array}{c}\text { Restrictions on road and } \\
\text { telecommunications access } \\
\text { between the village and the } \\
\text { protected area }\end{array}$ \\
\hline Stable environmental legislation & $\begin{array}{l}\text { Tourism promotion with the } \\
\text { Ministry of Tourism campaign All } \\
\text { you need is Ecuador }\end{array}$ & & $\begin{array}{l}\text { Increase in social problems due } \\
\text { to lack of jobs, medical services, } \\
\text { family planning and (high school } \\
\text { and higher) education }\end{array}$ \\
\hline $\begin{array}{l}\text { Availability of land for the crea- } \\
\text { tion of new infrastructure }\end{array}$ & $\begin{array}{l}\text { Current development of a } \\
\text { participatory management plan } \\
\text { for Loma Alta }\end{array}$ & $\begin{array}{l}\text { Lack of solidarity among } \\
\text { community members }\end{array}$ & $\begin{array}{l}\text { Conflicts of interest of certain } \\
\text { social actors }\end{array}$ \\
\hline
\end{tabular}

Source: Prepared by the authors. 
resources be updated and that the mechanisms for ensuring compliance be specified.

At the administrative level, the creation of an administrative unit with qualified staff will encourage participation and enhance the economy at the local level. The unit must have a director, technicians, assistants and park rangers until it is consolidated and expanded according to its needs.

At the socio-educational level, social participation and environmental education with diversity, inclusion and equity criteria that empower actors and sectors for proper planning and management of resources are crucial. The development of multi- and transdisciplinary scientific research through longterm projects and monitoring programs is essential to support decision-making.

At the socio-political level, governance follows a co-management model between the public administration represented by the Ministry of the Environment of Ecuador (MAE, by its Spanish acronym) through their respective directorates, the decentralized autonomous governments (e.g., governorate and municipality), and the local administration constituted by the cabildo in each community. However, it lacks a comprehensive territorial plan that incorporates the concept of sustainability with horizontal, systematized and multilevel social interactions. There is, therefore, a need for re-engineering processes of participatory environmental management that is unified into local, national and regional policy.

At the economic level, there is an urgent need to diversify financial mechanisms in order to integrate them into the process of change in the productive matrix focused on agriculture. Innovative financial mechanisms based on the proposed environmental services relate to payment for use or impact on environmental and cultural services, as well as management of biodiversity and non-timber resources.

In addition, a total of eight conservation targets (four natural and four cultural) were identified in the workshops, coinciding in both communities and with the preliminary list of experts. The natural conservation targets (NCT) were: 1) garúa forest; 2) hydrological systems; 3) game species, the white-tailed deer (Odocoileus virginianus), saíno (Tayassu tajaco), paca (Agouti paca), and Central American agouti (Dasyprocta punctata) were included in this category, since there is a tradition of consumptive local use of these species; and, 4) avifauna, the Esmeraldas woodstar (Chaetocercus berlepschi) and the yellow-throated toucan (Ramphastos ambiguus) were included in this category.
Due to the educational level of most participants, the coarse/fine filter strategy was adapted combining biological levels of biodiversity (ecosystems, communities and species) and conservation levels (coarse/fine filter) for the explanation of concepts and dissemination of results in the community (see Figure 2). An example with the NCT most valued by the community at the coarse filter level considering the geographical scale was shown (see Figure 3).

Regarding the four cultural conservation targets, the chosen ones were: 1) gastronomy, the inhabitants described typical dishes such as the seco de guanta (paca stew), seco de venado (deer stew) and the ceviche de chumumo as the most popular in the communities; 2) patron saint festivities, being the foundation of the community, the Virgen de las Mercedes Day and the Day of the Dead the most celebrated; and, 3) archaeological remains (huacas). The fourth target always fluctuated between the building (e.g. community center), collective memory (e.g. history of the community), the social institution (e.g. community) and local knowledge and practices (e.g. production of handicrafts using tagua and toquilla straw) (Figure 4); of these, the latter is almost non-existent within the community of Loma Alta.

\section{DISCUSSION}

The conservation design methodology has been used by academic entities, governmental and non-governmental organizations in the USA and Latin America, as part of ecoregional planning. This methodology has been suitable for this type of research and the area, as indicated by Chávez et al. (2014), is quantitative, as it uses ecological, biophysical, social criteria, applied to large geographical areas and all types of ecosystems, and is therefore considered to be systematic, efficient, replicable and participatory.

The results of the SWOT and the proposed measures are inputs for the development of a local strategy that strengthens integrated territorial management and have been obtained from a transdisciplinary knowledge, which reflects the dynamics in resource management and organizations. Thus, the results of the Conservation Action Planning (CAP) focused on natural conservation targets chosen by the community members are related to the list previously prepared with the experts, because they both agree that the humid forest, known as garúa forest beginning at 400 masl, must be preserved. The conservation of these forests is imperative because they provide taxonomic diversity and environmental 

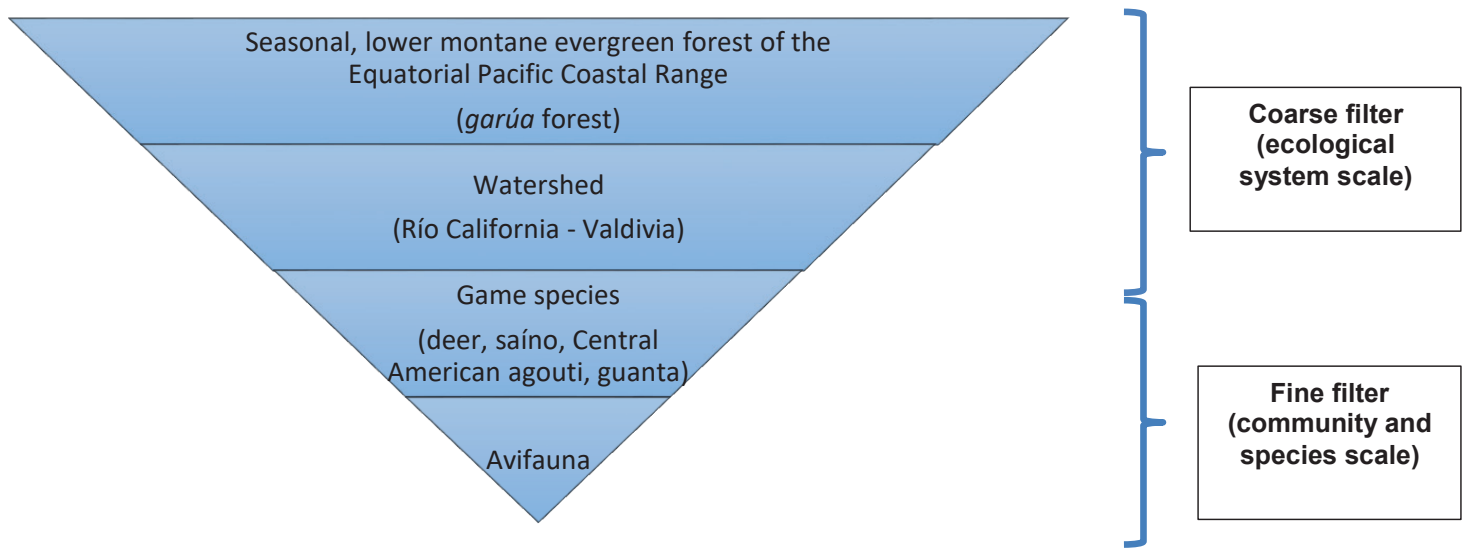

Figure 2. Natural conservation targets (NCT) adapted using coarse/fine filter technique. Source: Prepared by the authors.

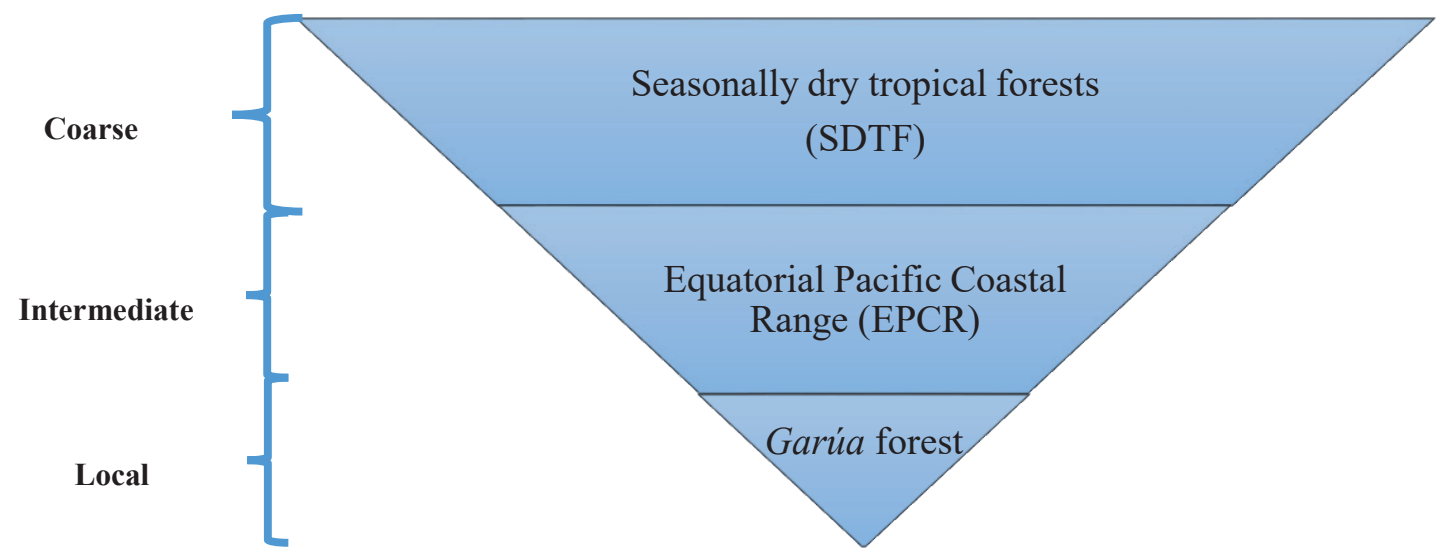

Figure 3. Terrestrial conservation target adapted using coarse filter.

Source: Prepared by the authors.

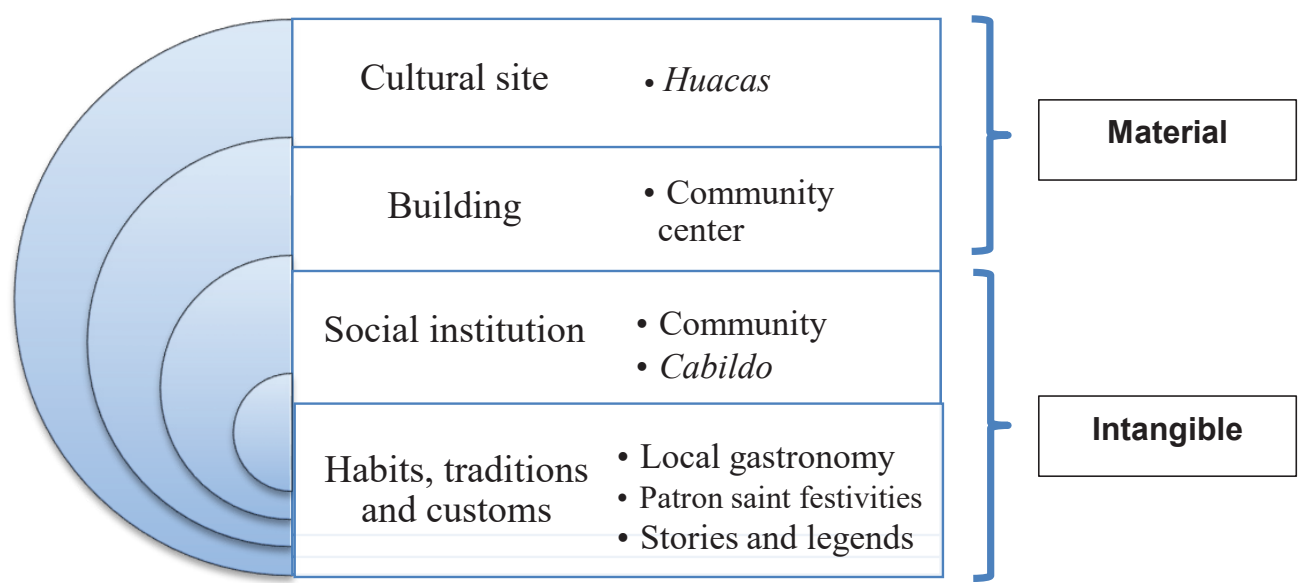

Figure 4. Cultural conservation targets.

Source: Prepared by the authors. 
services for local human survival in an area characterized by dry climate and xerophytic vegetation. Therefore, the conservation of these forests in the PSE must be a priority in the national, provincial and local agenda, to ensure these two communities continue protecting the remaining, diverse, representative and continuous flora.

In this way, biodiversity conservation needs up-todate scientific information for the design of regional development strategies and policies, as well as for the development of plans of conservation and sustainable use of biodiversity (Alvarado et al., 2011). According to Zamora (2002), descriptive scientific research is a basic and fundamental contribution to managing nature; ecological processes and their mechanisms should be analyzed in order to make better decisions. The results of this research therefore enable the improvement of environmental management by knowing the values in the PSE' forest reserve to be preserved, because the communities lack updated biological information and planning tools for efficient management.

Sustainable management, according to the Millennium Ecosystem Assessment (2005), requires a substantial change within institutions and governance, And their policies to reduce the severity of the issued faced by ecosystems. The democratization of environmental issues, based on the new Constitution of the Republic of Ecuador, has represented a paradigm shift in the conservation and management of biodiversity, which has promoted efficient environmental management and participatory governance.

In the PES, participatory governance is a primary factor in the management of resources due to indigenous-rural cultural identity and the biocentric and holistic worldview of rural communities. Loma Alta led the conservation process in the CCR, which, according to Astudillo et al. (2015), began in 1987, and protects an area of 1858.25 hectares of its forest. In addition, conservation through use has had successful experiences in indigenous communities; an example of this also occurred in Loma Alta, where Becker et al. (2005) demonstrated the potential of social participation in governance processes, which through long-term scientific research managed to preserve biodiversity and establish local and national institutional nesting. Other examples of participatory governance and conservation through use are seen in the province of Napo, in the Sumaco Biosphere Reserve (Torres et al., 2013), and in Honduras and Mexico, in the Mesoamerican tropical dry forest (Barrance et al., 2009).
The results of Becker et al. (2005) agree with the research by Ostrom (2010) on the Communities who focused on social capital and collective action in the use of natural capital, and polycentric governance through multi-level institutional analysis. Based on these previous investigations, we continued with the processes of participatory governance and strengthening the institutional and multilevel nesting in the development of this project, which is reflected in the results, where it was associated with academia (e.g., Universidad de Especialidades Espirítu Santo), local authorities (e.g., Loma Alta and Dos Mangas' cabildos, Municipality of Santa Elena) and national authorities (e.g., Ministry of Environment and Ministry of Tourism).

Despite the progress in the conservation of their resources and the annual monetary incentive that the municipalities receive from the Socio Bosque program, it is necessary to integrate these protected areas into regional and local development, where the biodiversity conservation perspective is integrated into eco-regional planning and land management. One planning and conservation strategy that has been promoted since the last two decades of the last century is territorial ecological connectivity, which maintains a functional connection derived from the motor skills of species to maintain ecological flows at landscape scale (EUROPARC-España, 2009).

In planning strategies, conservation corridors address ecological connectivity. Conservation corridors are management tools that combine human development needs with conservation priorities; their function is to allow the connection between protected areas or fragments of habitats to maintain the sustainability of natural ecological processes and to increase mobility and genetic exchange between species (Critical Ecosystem Partnership Fund, 2005). Within the Chocó-Darién-Western Ecuador Terrestrial Ecoregion, the Chocó-Manabí Conservation Corridor was created, which has a conservation strategy and establishes strategic alliances between Ecuador-Colombia and the United States.

Despite this initiative, the PSE and its protected areas were not included in the development of this strategy. The results of Astudillo et al. (2019a, b). on the analysis of spatial arrangement of plant formations and floristic similarity in several localities of the CCR emphasizes the plant affinity by altitudinal gradient and not by area, which reinforces the concept of ecological corridor. Moreover, the results of this research highlight the efforts of local and national social actors to promote projects that 
strengthen their capacities to plan and manage their natural and cultural resources. In terms of tourism planning, Chóez et al. (2017) proposed scenic trail that runs through the two communities to promote environmental and human sustainability. However, this initiative has been unsuccessful due to a lack of organization in both communities, deficiencies in the management of their resources and a deficit in long-term strategic planning.

\section{CONCLUSIONS}

1. The efficient management of seasonally dry tropical forests located in the PSE is faced with the challenge of strengthening local environmental planning and management capacities.

2. The management of natural and cultural resources in Santa Elena faces technical and administrative shortcomings due to the lack of a management plan and an autonomous administrative department to manage socio-environmental processes in protected areas and promote sustainable development through the diversification of innovative financial mechanisms based on environmental services.

3. The recognition of the forest and the social institutionality of the cabildo as natural and cultural conservation targets strengthen the concept of conservation through the use of resources that operates at the communal level for Santa Elena, but it must be consistent with local conditions of culture, land tenure and local development needs.

4. The current environmental governance in the PSE should reinforce participatory, inclusive, informed and fair management methods in order to achieve the sustainability of protected areas.

\section{ACKNOWLEDGEMENTS}

To the Research Center of the Universidad de Especialidades Espíritu Santo (UEES) for funding the execution of this project (UEES-2014-ART-002); to the deans, professors and pupils of the School of Liberal Arts and Education Sciences (Departments of Environmental Sciences, Psychology and Education), Economics (Department of Tourism) and Communication (Department of Design and Communication). Also, to the Ministry of the Environment of Ecuador (MAE), through its Provincial Directorate of Santa Elena for the corresponding research permit No. 017-15 IC-FAU-DPSE-MA, to the Municipality of Santa Elena and to Dionicio Gonzabay, Mayor of Santa Elena. To the local authorities, Gustavo de la A (President of Loma Alta) and Ángel Merchán (President of Dos Mangas) and the park rangers who contributed to the realization of this project.

\section{REFERENCES}

[1] Acción en el Biocorredor (2012). Plan de acción del Biocorredor territorio Chongón Colonche: biocorredores para el buen vivir. Guayaquil, Ecuador: FIDES, SGP, GEF y UNDP.

[2] Aguirre, N. \& Erazo, A. (2017). Valoración económica ambiental del compartimiento leñoso como una alternativa para conservar la biodiversidad del bosque seco de la provincia de Loja, Ecuador. Bosques Latitud Cero, 7(1), 89-107.

[3] Altmann, P. (2013). El Sumak Kawsay en el discurso del movimiento indígena ecuatoriano. Indiana, 30, 283-299. Retrieved from https://www.iai.spk-berlin.de/fileadmin/ dokumentenbibliothek/Indiana/Indiana_30/ IND_30_2013_283-299_Altmann.pdf.

[4] Alvarado, J.; Herrera, B.; Corrales, L.; Asch, J. \& Paaby, P. (2011). Identificación de las prioridades de conservación de la biodiversidad marina y costera en Costa Rica. Revista de Biología Tropical, 59(2), 829-842. Retrieved from https://revistas.ucr.ac.cr/index.php/rbt/ article/view/3143/3050.

[5] Astudillo, E. (2010). El desarrollo sostenible comunitario en un ENP de alto interés científico: el caso de la comuna Loma Alta y su reserva ecológica. (Master thesis). Universidad Autónoma de Madrid, Madrid.

[6] Astudillo-Sánchez, E., Pérez, J., Troccoli, L., Aponte, H., \& Tinoco, O. (2019b). Flora Leñosa del Bosque de Garúa de la Cordillera Chongón Colonche, Santa Elena-Ecuador. Ecología Aplicada, 18(2), 155-169. http://dx.doi. org/10.21704/rea.v18i2.1334

[7] Astudillo, E.; Pérez, J. \& Fabara, M. (2015). Árboles y arbustos: una relación con la avifauna. Samborondón, Ecuador: Universidad Espíritu Santo.

[8] Astudillo-Sánchez, E., Pérez, J., Troccoli, L., \& Aponte, H. (2019a). Composición, estructura y diversidad vegetal de la Reserva Ecológica Comunal Loma Alta, Santa Elena, Ecuador. Revista Mexicana de Biodiversidad, 90(1), 1-25. https://doi.org/10.22201/ ib.20078706e.2019.90.2871 
[9] Barrance, A.; Schreckenberg, K. \& Gordon, J. (2009). Conservación mediante el uso: lecciones aprendidas en el bosque seco tropical mesoamericano. London, England: Overseas Development Institute.

[10] Becker, D. (1999). Protecting a garúa forest in Ecuador: the role of institutions and ecosystem valuation. Ambio, 28(2), 156-161. Retrieved from https://lifenetnature.org/download/docs/ lifenetnature-org_protecting-garua-forest.pdf.

[11] Becker, D.; Agreda, A.; Astudillo, E.; Costantino, M. \& Torres, P. (2005). Community-based monitoring of fog capture and biodiversity at Loma Alta, Ecuador: enhanced social capital and institutional cooperation. Biodiversity and Conservation, 14, 2695-2707. Retrieved from http://www.monitoringmatters.org/articles/ full9.pdf.

[12] Best, B. \& Kessler, M. (1995). Biodiversity and conservation in tumbesian Ecuador and Peru. Cambridge, Inglaterra: BirdLife International. Retrieved from https://pavaaliblanca.weebly. com/uploads/5/0/3/0/50301069/best_kessler. pdf.

[13] Chávez, H.; González, M. \& Hernández, P. (2014). Metodologías para identificar áreas prioritarias para conservación de ecosistemas naturales. Revista Mexicana de Ciencias Forestales, 6(27), 8-23. Retrieved from http:// www.scielo.org.mx/pdf/remcf/v6n27/v6n27a2. pdf.

[14] Chóez, M.; Dávila, C. \& Astudillo, E. (2017). Diagnóstico del potencial emprendedor y turístico en la comuna Loma Alta, Santa Elena. Samborondón, Ecuador: Universidad Espíritu Santo.

[15] Cifuentes, M.; Izurieta, A. \& Henrique, H. (2000). Medición de la efectividad del manejo de áreas protegidas. Turrialba, Costa Rica: World Wildlife Fund.

[16] Columba, K. (2013). Manual para la gestión operativa de las áreas protegidas del Ecuador. Quito, Ecuador: Ministerio del Medio Ambiente I Punto Verde.

[17] Critical Ecosystem Partnership Fund (2005). Perfil del ecosistema: Corredor de Conservación Chocó-Manabí, ecorregión terrestre prioritaria del Corredor Chocó-DarienEcuador occidental (hotspot). Colombia y Ecuador. Retrieved from https://www.cepf.net/ sites/default/files/final.spanish.choco-darienwestern-ecuador.choco_.ep_.pdf.
[18] EUROPARC-España (2009). Conectividad ecológica y áreas protegidas. Herramientas y casos prácticos. Madrid, Spain: FUNGOBE. http://www.redeuroparc.org/system/files/ shared/monografia2.pdf.

[19] Gobierno autónomo descentralizado municipal Santa Elena (2014). Plan de desarrollo y ordenamiento territorial-Cantón Santa Elena 2014-2019. Retrieved from http://app.sni. gob.ec/sni-link/sni/PORTAL_SNI/data_sigad_ plus/sigadplusdiagnostico/0960001540001_ PLAN\%20DE\%20DESARROLLO\%20Y\% 20 ORDENAMIENTO\%20TERRITORIAL \%203001-2015-2\%20fin_19-02-2015_09-41-20.pdf.

[20] Granizo, T.; Molina, M.; Secaira, E.; Herrera, B.; Benítez, S.; Maldonado, O.; Libby, M.; Arroyo, P.; Ísola, S. \& Castro, M. (2006). Manual de planificación para la conservación de áreas, $P C A$. Quito, Ecuador: The Nature Conservancy y The United States Agency for International Development.

[21] Groves, C.; Valutis, L.; Vosick, D.; Neely, B.; Wheaton, K.; Touval, J. \& Runnels, B. (2000). Diseño de una geografía de la esperanza. Manual para la planificación de la conservación ecorregional (vols. 1 y 2). Arlington, USA: The Nature Conservancy.

[22] Gurrutxaga, M. \& Lozano, P. (2009). La integración de la conectividad ecológica en los instrumentos de ordenación y planificación territorial: una revisión. Boletín de la Asociación de Geógrafos Españoles, (49), 45-66.

[23] López, E. \& Peralta, P. (2016). Breve síntesis histórica y reflexiones acerca de la continuidad étnica, sentido de etnicidad e identidad cultural en la región de Santa Elena, Ecuador. Revista Científica y Tecnológica UPSE, 3(3), 99-105.

[24] Millennium Ecosystem Assessment (2005). Ecosystems and human well-being. Synthesis. Washington, USA: Island Press.

[25] Ministerio del Ambiente del Ecuador (2012a). Línea base de deforestación del Ecuador continental. Quito, Ecuador: Ministerio del Ambiente del Ecuador / Socio Bosque.

[26] Ministerio del Ambiente del Ecuador (2012b). Sistema de clasificación de los ecosistemas del Ecuador continental. Quito, Ecuador: Subsecretaría de Patrimonio Natural.

[27] Ministerio del Ambiente del Ecuador (2017). Análisis de vulnerabilidad local al cambio climático del sector ganadero en las zonas de implementación del proyecto $\mathrm{MGCl}$ en la 
provincia de Santa Elena. Quito, Ecuador: Ministerio de Agricultura y Ganadería.

[28] Organización de las Naciones Unidas para la Alimentación (2016). El estado de los bosques del mundo 2016. Los bosques y la agricultura: desafíos y oportunidades en relación con el uso de la tierra. Roma, Italia: Food and Agriculture Organization of the United Nations (FAO). Retrieved from http://www.fao.org/3/i5588s/ i5588s.pdf.

[29] Ostrom, E. (2010). Polycentric systems for coping with collective action and global environmental change. Global Environmental Change, 20(4), 550-557.

[30] Ponce, H. (2006). La matriz FODA: una alternativa para realizar diagnósticos y determinar estrategias de intervención en las organizaciones productivas y sociales. Contribuciones a la Economía, 2, 1-16.
[31] Thompson, A. A.; Strickland, A. J.; Colado, P. I. \& Carreón, M. A. S. (1998). Dirección y administración estratégicas: conceptos, casos y lecturas. México, D. F., Mexico: McGraw-Hill.

[32] Torres, B.; Starnfeld, F.; Vargas, J.; Ramm, G.; Chapalbay, R.; Rios, M.; Gómez, A.; Torricelli, Y.; Jurrius, I.; Tapia, A.; Shiguango, J.; Torres, A. Velasco, C.; Murgueytio, A. y CordobaBahle, D. (2013). Gobernanza participativa en la Amazonía del Ecuador: recursos naturales y desarrollo sostenible. Puyo, Ecuador: Universidad Estatal Amazónica, Ministerio del Ambiente del Ecuador, Gobierno Autónomo Descentralizado Provincial de Napo y Cooperación Alemana al Desarrollo.

[33] Zamora, R. (2002). Los espacios protegidos necesitan una gestión activa. Quercus, 191, 64-65. 